\title{
Credibilistic Loss Aversion Nash Equilibrium for Bimatrix Games with Triangular Fuzzy Payoffs
}

\author{
Chunsheng Cui $\mathbb{D}^{1},{ }^{1}$ Zhongwei Feng $\mathbb{D}^{2},{ }^{2}$ and Chunqiao Tan $\mathbb{D}^{3}$ \\ ${ }^{1}$ School of Information, Beijing Wuzi University, Beijing 101149, China \\ ${ }^{2}$ School of Business, Central South University, Changsha 410083, China \\ ${ }^{3}$ School of Government Audit, Nanjing Audit University, Nanjing 211815, China \\ Correspondence should be addressed to Zhongwei Feng; 1064537995@qq.com and Chunqiao Tan; chunqiaot@sina.com
}

Received 24 July 2018; Revised 26 October 2018; Accepted 7 November 2018; Published 16 December 2018

Academic Editor: Hassan Zargarzadeh

Copyright (C) 2018 Chunsheng Cui et al. This is an open access article distributed under the Creative Commons Attribution License, which permits unrestricted use, distribution, and reproduction in any medium, provided the original work is properly cited.

\begin{abstract}
Inspired by Shalev's model of loss aversion, we investigate the effect of loss aversion on a bimatrix game where the payoffs in the bimatrix game are characterized by triangular fuzzy variables. First, we define three solution concepts of credibilistic loss aversion Nash equilibria, and their existence theorems are presented. Then, three sufficient and necessary conditions are given to find the credibilistic loss aversion Nash equilibria. Moreover, the relationship among the three credibilistic loss aversion Nash equilibria is discussed in detail. Finally, for $2 \times 2$ bimatix game with triangular fuzzy payoffs, we investigate the effect of loss aversion coefficients and confidence levels on the three credibilistic loss aversion Nash equilibria. It is found that an increase of loss aversion levels of a player leads to a decrease of his/her own payoff. We also find that the equilibrium utilities of players are decreasing (increasing) as their own confidence levels when players employ the optimistic (pessimistic) value criterion.
\end{abstract}

\section{Introduction}

Since established by von Neumann and Morgenstern (1944), game theory has been applied to modeling strategic interactions for decision problem in many fields, such as economics, political science, and psychology. A bimatrix game is a finite noncooperative game in normal (or strategic) form, which is often used to analyze interaction between two rational players who behave strategically. In this game, each player desires achieving as much payoffs as possible by choosing one from her/his pure strategy set. A bimatrix game is modeled by two payoff matrices determined by the two players' strategic profiles. A mixed strategy describes a situation that each player would rather randomly choose a pure strategy with a given probability distribution than choose a particular pure strategy. Nash [1] proved that there exists at least one mixed strategy Nash equilibrium such that no player can improve her/his gains by unilaterally deviating from such strategy profiles.

As a matter of fact, uncertainty for parameters plays an important role in bimatrix games, which is often called incomplete information. The most striking style of the uncertainty for parameters is the uncertainty for the payoffs, which includes random payoffs and fuzzy payoffs. As pointed out by Harsanyi [2], each player often lacks the information about her/his opponents' or even her/his own payoffs. In order to deal with games with incomplete information, Harsanyi [2] proposed Bayesian game model that is often used to deal with games with random payoffs. Since then the game with random payoffs has been investigated by many researchers [3-8]. When it comes to games with fuzzy payoffs, the Bayesian game model fails to be applied to such games. Fuzzy set theory provides an efficient alternative to model the uncertainty about payoffs that are modeled by fuzzy variables. Campos [9] firstly investigated two-person zero-sum games with fuzzy payoffs. Since then two-person games with fuzzy payoffs, including two-person zero-sum games and bimatrix games, have been investigated by many researchers [10-22]. Moreover, some credibilistic games are proposed to deal with the games with fuzzy payoffs, including credibilistic strategic game [23-26] and credibilistic coalitional game [27]. 
It is worthwhile noting that the existing literature on games with fuzzy payoffs ignores the effect of decisionmaker's behavior characteristics on equilibrium strategies. In real game problems, players often show bounded rational behavior characteristics. A number of experimental works in the psychological and the economic literature suggest that decision-makers are motivated to minimize losses (relative to a reference point) much more than they are motivated to maximize gains [28-32]. In other words, decision-makers are loss-averse. Players' loss aversion plays an important role in real game problems. For example, Langsha Group, the largest socks manufacturer of China, decided to terminate cooperation with Wal-Mart in 2007, since Langsha Group could no longer benefit from cooperation with Wal-Mart. That is, Wal-Mart grabbed too much profit to make Langsha Group incur losses. Thus, to incorporate loss aversion into games, Shalev [33] proposed an elegant and simple model of loss aversion to measure loss aversion: the utility of an outcome below the reference point is obtained from the basic utility by subtracting a disutility that is equal to the size of the loss multiplied by the loss aversion coefficient. Since then Shalev's model of loss aversion has received attention in the field of game theory based on the assumption that the payoffs are common knowledge to two players [34-38]. However, it is very likely that this assumption is violated, since the decision environment is often characterized by lots of strategies, complicated relations between strategic choices, and their intricate influences on payoffs, which leads to the uncertainty of the payoffs. For example, Langsha Group develops its direct selling business in 2017; it cannot be exactly predicted whether Langsha Group can benefit from the direct selling because of the uncertainty of the consumer demand and the investment cost in direct selling business. Thus, to deal with such situation faced by Langsha Group, this paper investigates the impact of loss aversion on equilibrium strategies in bimatrix games with fuzzy payoffs.

In this paper, we extend the analysis of bimatrix games with fuzzy payoffs to incorporate loss aversion. Inspired by Shalev's model of loss aversion, we first give a formula that relates outcomes and reference points to utility. This formula, where the parameters except loss aversion coefficient are modeled as triangular fuzzy variables, is called loss aversion utility function with triangular fuzzy parameters. Then, given an extended bimatrix game with triangular fuzzy payoffs, including a bimatrix game with triangular fuzzy payoffs and loss aversion coefficients, three ranking methods are used to model different situations. And we define the solution concepts of three credibilistic loss aversion equilibria and discuss the relationship among the three credibilistic loss aversion equilibria. Finally, by means of $2 \times 2$ bimatrix game with triangular fuzzy payoffs, we investigate the effect loss aversion coefficients and confidence levels on the three credibilistic loss aversion Nash equilibria.

The rest of this paper is organized as follows. In Section 2, credibility theory and loss aversion are briefly reviewed. In Section 3, the concepts of three credibilistic loss aversion Nash equilibria are proposed, and their existence theorems and sufficient and necessary conditions are given. Moreover, the relationship of the three credibilistic loss aversion Nash equilibria is discussed. In Section 4, we investigate the effect of loss aversion coefficients and confidence levels on the three credibilistic loss aversion Nash equilibria. In Section 5, conclusions are shown.

\section{Preliminaries}

2.1. Credibility Theory. As a branch of mathematics, credibility theory is often used to model the behavior of fuzzy phenomena. Since proposed by Liu [39], credibility theory has been used extensively in many fields, such as portfolio selection [40, 41] and transportation planning [42, 43].

Definition 1 (see [44]). Let $\Theta$ be a nonempty set and $\Xi(\Theta)$ be the power set of $\Theta$; a set function $\operatorname{Cr}\{\cdot\}$ is called a credibility measure if it satisfies the following four axioms.

Axiom 1 (normality). $\operatorname{Cr}\{\Theta\}=1$.

Axiom 2 (monotonicity). $C r\{A\} \leq C r\{B\}$, where $A \subset B$.

Axiom 3 (self-duality). $\operatorname{Cr}\{A\}+\operatorname{Cr}\left\{A^{c}\right\}=1$ for any $A \in \Xi(\Theta)$.

Axiom 4 (maximality). $\operatorname{Cr}\left\{\bigcup_{i} A_{i}\right\}=\sup _{i} \operatorname{Cr}\left\{A_{i}\right\}$ for any $\left\{A_{i}\right\}$ with $\sup _{i} \operatorname{Cr}\left\{A_{i}\right\}<0.5$.

Definition 2 (see [44]). Let $\Theta$ be a nonempty set, $\Xi(\Theta)$ be the power set of $\Theta$, and $C r$ be a credibility measure; then the triplet $(\Theta, \Xi(\Theta)$, Cr) is called a credibility space.

Definition 3 (see [44]). A fuzzy variable $\widetilde{v}$ is a measurable function from a credibility space $(\Theta, \Xi(\Theta), \mathrm{Cr})$ to the set of real numbers.

Lemma 4 (see [44]). Let $\widetilde{v}$ be a fuzzy variable with membership function $\mu$; then for any set $B$ of real numbers, we have

$$
C r\{\widetilde{v} \in B\}=\frac{1}{2}\left(\sup _{x \in B} \mu(x)+1-\sup _{x \notin B^{c}} \mu(x)\right)
$$

As a special fuzzy variable with the triangular fuzzy membership function $\mu(x)$, a triangular fuzzy variable is denoted by $\widetilde{v}=\left(v^{L}, v^{M}, v^{U}\right)$ [45], where $v^{M}$ is the mean of $\widetilde{v}$ and $v^{L}$ and $v^{U}$ are the low bound and the bound of $\widetilde{v}$, respectively. Its membership function is given by

$$
\mu(x)= \begin{cases}\frac{\left(x-v^{L}\right)}{\left(v^{M}-v^{L}\right)} & \text { if } v^{L} \leq x<v^{M} \\ 1 & \text { if } x=v^{M} \\ \frac{\left(v^{U}-x\right)}{\left(v^{U}-v^{M}\right)} & \text { if } v^{M}<x \leq v^{U} \\ 0 & \text { else }\end{cases}
$$

Obviously, if $v^{L}=v^{M}=v^{U}$, then the triangular fuzzy variable $\widetilde{v}=\left(v^{L}, v^{M}, v^{U}\right)$ is reduced to a real number. For a triangular fuzzy number $\widetilde{v}=\left(v^{L}, v^{M}, v^{U}\right)$, if $v^{L} \geq 0$ and $v^{U}>0$, we call $\widetilde{v}$ nonnegative triangular fuzzy number. Let $\widetilde{v}=\left(v^{L}, v^{M}, v^{U}\right)$ and $\widetilde{\eta}=\left(\eta^{L}, \eta^{M}, \eta^{U}\right)$ be two triangular 
fuzzy numbers; then according to extension principle proposed by Zadeh [46] and Negoita et al. [47], we have the following arithmetical operations:

(i) $\tilde{v}+\widetilde{\eta}=\left(v^{L}+\eta^{L}, v^{M}+\eta^{M}, v^{U}+\eta^{U}\right)$;

(ii) $\widetilde{v}-\widetilde{\eta}=\left(v^{L}-\eta^{U}, v^{M}-\eta^{M}, v^{U}-\eta^{L}\right)$;

(iii) $a \widetilde{v}=\left(a v^{L}, a v^{M}, a v^{U}\right)$ if $a \geq 0$.

It follows from extension principle that $\widetilde{v}-\widetilde{v} \neq(0,0,0)=0$. However, in optimization decision problems, it can be desirable to have crisp values for $\widetilde{v}-\widetilde{v}=0$. In order to deal with such situations, Gani and Assarudeen [48] developed a new operation for subtraction on triangular fuzzy numbers.

Lemma 5 (see [48]). Let $\widetilde{v}=\left(v^{L}, v^{M}, v^{U}\right)$ and $\tilde{\eta}=\left(\eta^{L}, \eta^{M}\right.$, $\left.\eta^{U}\right)$ be two triangular fuzzy numbers; then we have

$$
\widetilde{v}-\tilde{\eta}=\left(v^{L}-\eta^{L}, v^{M}-\eta^{M}, v^{U}-\eta^{U}\right)
$$

Definition 6 (see [44]). The fuzzy variables $\widetilde{v}_{1}, \widetilde{v}_{2}, \ldots, \widetilde{v}_{m}$ are said to be independent if

$$
\operatorname{Cr}\left\{\bigcap_{i=1}^{m}\left(\widetilde{v}_{i} \in B_{i}\right)\right\}=\min _{1 \leq i \leq m} \operatorname{Cr}\left\{\widetilde{v}_{i} \in B_{i}\right\}
$$

for any sets $B_{1}, B_{2}, \ldots, B_{m}$ of $R$.

Definition 7 (see [49]). Let $\widetilde{v}$ be a fuzzy variable; then the expected value of $\widetilde{v}$ is defined by

$$
E[\widetilde{v}]=\int_{0}^{+\infty} \operatorname{Cr}\{\tilde{v} \geq r\} d r-\int_{-\infty}^{0} C r\{\widetilde{v} \leq r\} d r
$$

provided that at least one of the two integrals is finite.

Lemma 8 (see [50]). Let $\widetilde{v}$ and $\widetilde{\eta}$ be independent fuzzy variables with finite expected values; then for any numbers a and $b$, we have

$$
E[a \widetilde{v}+b \widetilde{\eta}]=a E[\widetilde{v}]+b E[\widetilde{\eta}]
$$

Definition 9 (see [50]). Let $\widetilde{v}$ be a fuzzy variable and $\alpha \in(0,1]$ be a confidence level; then, for a real number $r$,

$$
\widetilde{v}_{\text {sup }}(\alpha)=\sup \{r \mid C r\{\widetilde{v} \geq r\} \geq \alpha\}
$$

is called $\alpha$-optimistic value to $\widetilde{v}$, and

$$
\widetilde{v}_{\text {inf }}(\alpha)=\inf \{r \mid \operatorname{Cr}\{\widetilde{v} \leq r\} \geq \alpha\}
$$

is called $\alpha$-pessimistic value to $\widetilde{v}$.

This means that the fuzzy variable $\widetilde{v}$ will reach upwards of the $\alpha$-optimistic value $\widetilde{v}_{\text {sup }}(\alpha)$ with credibility $\alpha$ and will be below the $\alpha$-pessimistic value $\widetilde{v}_{\text {inf }}(\alpha)$ with credibility $\alpha$. In other words, the $\alpha$-optimistic value $\widetilde{v}_{\text {sup }}(\alpha)$ is the supremum value that $\widetilde{v}$ achieves with credibility $\alpha$, and the $\alpha$-pessimistic value $\widetilde{v}_{\text {inf }}(\alpha)$ is the infimum value that $\widetilde{v}$ achieves with credibility $\alpha$.
Lemma 10 (see [50]). Let $\widetilde{v}$ and $\tilde{\eta}$ be independent fuzzy variables; then for any $\alpha \in(0,1]$ and any nonnegative numbers $a$ and $b$, we have

$$
\begin{aligned}
& (a \widetilde{v}+b \widetilde{\eta})_{\text {sup }}(\alpha)=a \widetilde{v}_{\text {sup }}(\alpha)+b \widetilde{\eta}_{\text {sup }}(\alpha) \\
& (a \widetilde{v}+b \widetilde{\eta})_{\mathrm{inf}}(\alpha)=a \widetilde{v}_{\mathrm{inf}}(\alpha)+b \widetilde{\eta}_{\mathrm{inf}}(\alpha)
\end{aligned}
$$

Lemma 11 (Liu and Liu, 2007). Let $\widetilde{v}$ be a fuzzy variable; then for any $\alpha \in(0,1]$, we have

$$
\begin{aligned}
\text { if } a & \geq 0, \\
\text { then }(a \widetilde{v})_{\text {sup }}(\alpha) & =a \widetilde{v}_{\text {sup }}(\alpha), \\
(a \widetilde{v})_{\text {inf }}(\alpha) & =a \widetilde{v}_{\text {inf }}(\alpha)
\end{aligned}
$$

In order to rank fuzzy variables, the following methods are often used.

Definition 12 (see [51]). Let $\widetilde{v}$ and $\widetilde{\eta}$ be independent fuzzy variables; then we have $E[\tilde{\eta}]$

(1) expected value criterion: $\widetilde{v}<\widetilde{\eta}$ if and only if $E[\widetilde{v}]<$

(2) optimistic value criterion: $\widetilde{v}<\widetilde{\eta}$ if and only if $\widetilde{v}_{\text {sup }}(\alpha)<$ $\tilde{\eta}_{\text {sup }}(\alpha)$ for some predetermined confidence level $\alpha \in(0,1]$;

(3) pessimistic value criterion: $\widetilde{v}<\widetilde{\eta}$ if and only if $\widetilde{v}_{\text {inf }}(\alpha)<\widetilde{\eta}_{\text {inf }}(\alpha)$ for some predetermined confidence level $\alpha \in(0,1]$.

The expected value criterion is used to deal with the situation where a player wants to optimize the expected value of her/his payoff. The optimistic value criterion is applied to coping with the situation in which a player strives to optimize the optimistic value of her/his payoff at given a confidence level $\alpha$. The pessimistic value criterion is used to model the situation that a player strives to optimize the pessimistic value of her/his payoff at given a confidence level $\alpha$.

Let $\widetilde{v}_{1}, \widetilde{v}_{2}, \ldots, \widetilde{v}_{n}$ be independent fuzzy variables; then $\widetilde{v}=$ $\widetilde{v}_{1} \vee \widetilde{v}_{2} \vee \cdots \vee \widetilde{v}_{n}$ is called the maximum of $\widetilde{v}_{1}, \widetilde{v}_{2}, \ldots, \widetilde{v}_{n}$, and $\widetilde{v}=\widetilde{v}_{1} \wedge \widetilde{v}_{2} \wedge \cdots \wedge \widetilde{v}_{n}$ is called the minimum of $\widetilde{v}_{1}, \widetilde{v}_{2}, \ldots, \widetilde{v}_{n}$.

2.2. Loss Aversion. Kahneman and Tversky [29] first proposed loss aversion; its central assumption is that gains are smaller than losses. For instance, the decrease in utility of loss 10 dollars if one has 100 dollars is larger than the increase in utility of gain 10 dollars if one has 90 dollars. Shalev [33] proposed an elegant and simple model of loss aversion to measure this. The utility function is given by the following transformation

$$
u\left(w, w_{0}, \lambda\right)= \begin{cases}w & \text { if } w>w_{0} \\ w-\lambda\left(w_{0}-w\right) & \text { if } w<w_{0}\end{cases}
$$

or equivalently

$$
u\left(w, w_{0}, \lambda\right)=(1+\lambda) w-\lambda \max \left\{w_{0}, w\right\}
$$

where $\lambda$ is nonnegative and $w_{0}$ is a reference point. Shalev's model of loss aversion is similar to the value function given by Tversky and Kahneman [32]. The loss aversion aspect of the 
utility function is retained, which is that the marginal utility in losses is larger than in gains. Moreover, in order to reflect heterogeneity of loss aversion, the loss aversion coefficients are different for different players.

\section{Credibilistic Loss Aversion Nash Equilibria}

In this section, according to three decision criteria from credibility theory, i.e., expected value criterion, optimistic value criterion, and pessimistic value criterion, three credibilistic loss aversion Nash equilibria are proposed.

3.1. Bimatrix Game with Triangular Fuzzy Payoffs and Loss Aversion. Let $I$ and $J$ be two players and $M=\{1,2, \ldots, m\}$ and $N=\{1,2, \ldots, n\}$ be the pure strategy sets of players $I$ and $J$, respectively. The mixed strategy set of player $I$ is denoted by

$$
\begin{aligned}
S_{I} & =\left\{x=\left(x_{1}, x_{2}, \ldots, x_{m}\right)^{T} \in R_{+}^{m} \mid x_{i} \geq 0, i\right. \\
& \left.=1,2, \ldots, m, \sum_{i=1}^{m} x_{i}=1\right\},
\end{aligned}
$$

and the mixed strategy set of player $J$ is denoted by

$$
\begin{aligned}
S_{J} & =\left\{y=\left(y_{1}, y_{2}, \ldots, y_{n}\right)^{T} \in R_{+}^{n} \mid y_{j} \geq 0, j\right. \\
& \left.=1,2, \ldots, n, \sum_{j=1}^{n} y_{j}=1\right\} .
\end{aligned}
$$

A bimatrix game is denoted by $G_{1}=\left\langle\{I, J\}, S_{I} \times S_{J}, V, \Gamma\right\rangle$, where $V$ and $\Gamma$ are $m \times n$ payoff matrices of players $I$ and $J$, whose $i, j$ elements are $v_{i j}$ and $\eta_{i j}$. For any mixed strategy profile $(x, y)$, it determines the outcomes of the bimatrix game $\left(x^{T} V y, x^{T} \Gamma y\right)$, where $x^{T} V y$ and $x^{T} \Gamma y$ are expected payoffs of players $I$ and $J$, respectively.

Lemma 13 (see [1]). There exists at least one mixed strategy Nash equilibrium in a finite bimatrix game.

Since the decision environment is often characterized by lots of strategies, intricate relations between strategic choices and their influences on players' payoffs, it is impossible that players make accurate or probabilistic estimation of the payoff matrices. Thus, we consider a bimatrix game with fuzzy payoffs. Here, players' payoffs are modeled by triangular fuzzy variables. Triangular fuzzy numbers $\widetilde{v}_{i j}=\left(v_{i j}^{L}, v_{i j}^{M}, v_{i j}^{U}\right)$ and $\tilde{\eta}_{i j}=\left(\eta_{i j}^{L}, \eta_{i j}^{M}, \eta_{i j}^{U}\right)$ are denoted by the payoffs that players $I$ and $J$ obtain when the pure strategy profile $(i, j)$ is played. This bimatrix game with triangular fuzzy payoffs is denoted by $G_{2}=\left\langle\{I, J\}, S_{I} \times S_{J}, \widetilde{V}, \widetilde{\Gamma}\right\rangle$, where $\widetilde{V}$ and $\widetilde{\Gamma}$ are $m \times n$ payoff matrices of players $I$ and $J$, whose $i, j$ elements are $\widetilde{v}_{i j}$ and $\widetilde{\eta}_{i j}$. Furthermore, we assume that $\widetilde{v}_{i j}$ and $\widetilde{\eta}_{i j}$ are independent triangular fuzzy variables for all pure strategy profiles $(i, j)$.
In many situations, decision-makers are motivated to minimize losses (relative to a reference point) much more than they are motivated to maximize gains. We extend the analysis of the bimatrix game with fuzzy payoffs to incorporate loss aversion. An extended bimatrix game with triangular fuzzy payoffs $G_{3}=\left\langle\{I, J\}, S_{I} \times S_{I}, \widetilde{V}^{\lambda_{I}}, \widetilde{\Gamma}^{\lambda_{I}}\right\rangle$ has two additional elements - the loss aversion coefficients of players $I$ and $J$. Let $\lambda_{I} \in R_{+}$and $\lambda_{J} \in R_{+}$specify the levels of loss aversion of players $I$ and $J$, respectively. The higher the values of $\lambda_{I}$ and $\lambda_{J}$ are, the higher the degrees of loss aversion of players $I$ and $J$ are. Players $I$ and $J$ seek maximum of gains if $\lambda_{I}=0$ and $\lambda_{J}=0$, which means that the utility functions of players $I$ and $J$ are not reference dependent. Inspired by (13), let $\widetilde{v}_{I r}=\left(v_{I r}^{L}, v_{I r}^{M}, v_{I r}^{U}\right)$ be a reference point of player $I$ and $\widetilde{v}_{I r}$ and $\widetilde{v}_{i j}$ be independent triangular fuzzy variables. Given a reference point $\widetilde{v}_{I r}$ and a basic utility value $\widetilde{v}_{i j}$, then for player $I$, the final loss aversion utility is given by

$$
\widetilde{v}_{i j}^{\widetilde{v}_{I r}}=\left(1+\lambda_{I}\right) \widetilde{v}_{i j}-\lambda_{I}\left(\widetilde{v}_{i j} \vee \widetilde{v}_{I r}\right)
$$

Similarly, let $\widetilde{\eta}_{J r}=\left(\eta_{J r}^{L}, \eta_{J r}^{M}, \eta_{J r}^{U}\right)$ be a reference point of player $J$ and $\tilde{\eta}_{J r}$ and $\tilde{\eta}_{i j}$ be independent triangular fuzzy variables. Given a reference point $\widetilde{\eta}_{J r}$ and a basic utility value $\widetilde{\eta}_{i j}$, then for player $J$, the final loss aversion utility is given by

$$
\tilde{\eta}_{i j} \tilde{\eta}_{J r}=\left(1+\lambda_{J}\right) \tilde{\eta}_{i j}-\lambda_{J}\left(\tilde{\eta}_{i j} \vee \tilde{\eta}_{J r}\right)
$$

For exogenously given $\widetilde{v}_{I r}$ and $\widetilde{\eta}_{J r}$, an extended bimatrix game with triangular fuzzy payoffs can be transformed into a standard game with triangular fuzzy payoffs by evaluating the utility of each payoff according to (16) and (17). Given an extended bimatrix game $G_{3}$ and reference points $\widetilde{v}_{I r}$ and $\widetilde{\eta}_{J r}$, a transformation

$$
G_{4}=\left\langle\{I, J\}, S_{I} \times S_{J}, \widetilde{V}^{\lambda_{I}, \widetilde{v}_{I r}}, \widetilde{\Gamma}^{\lambda_{J}, \widetilde{\eta}_{J r}}\right\rangle
$$

is defined, where the utility of each payoff for each player is transformed by using the appropriate reference points and loss aversion coefficients according to (16) or (17).

For an extended bimatrix game with triangular fuzzy payoffs $G_{3}$, we define

$$
\begin{aligned}
& \underline{\underline{r}}=\min \left\{\min _{i \in M, j \in N} \widetilde{v}_{i j}, \min _{i \in M, j \in N} \widetilde{\eta}_{i j}\right\}, \\
& \overline{\widetilde{r}}=\max \left\{\max _{i \in M, j \in N} \widetilde{v}_{i j}, \max _{i \in M, j \in N} \widetilde{\eta}_{i j}\right\},
\end{aligned}
$$

where $\underline{\tilde{r}}$ and $\overline{\widetilde{r}}$ are the minimum and the maximum in matrices $\widetilde{V}$ and $\widetilde{\Gamma}$.

3.2. Definitions of Credibilistic Loss Aversion Nash Equilibrium Strategies. Suppose that the expected value criterion is employed. Given an extended bimatrix game $G_{3}$ and 
reference points $\widetilde{v}_{I r}$ and $\widetilde{\eta}_{J r}$, the loss aversion utilities of player $I$ and $J$ are given, respectively:

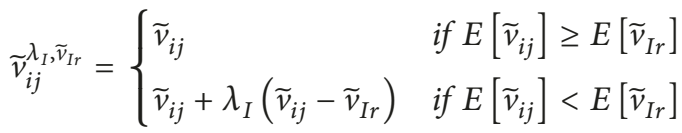

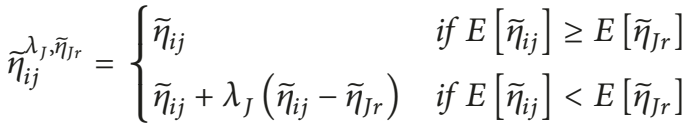

For a strategy profile $(x, y)$ and a reference point pair $\left(\widetilde{v}_{I r}, \widetilde{\eta}_{J r}\right)$, players $I$ and $J$ have expected final loss aversion utilities $x^{T} \widetilde{V}^{\lambda_{I}, \widetilde{v}_{I r}} y$ and $x^{T} \widetilde{\Gamma}^{\prime \lambda_{F}, \widetilde{\eta}_{I r}} y$, respectively. Note that the expected value criterion is used to deal with the situation that players want to maximize the expected values of their own expected final loss aversion utility. Thus, for player $I$, we have $E\left[x^{T} \widetilde{V}^{\lambda_{1}, \tilde{\underline{r}}} y\right]=E\left[x^{T} \widetilde{V} y\right] \geq E\left[\min _{i \in M, j \in N} \widetilde{v}_{i j}\right] \geq$ $E[\underline{\widetilde{r}}]$ and $E\left[x^{T} \widetilde{V}^{\lambda_{I}, \bar{r}} y\right] \leq E\left[x^{T} \widetilde{V} y\right] \leq E\left[\max _{i \in M, j \in N} \widetilde{v}_{i j}\right] \leq$ $E[\overline{\tilde{r}}]$. Since $E\left[x^{T} \widetilde{V}^{\lambda_{I}, \widetilde{v}_{I r}} y\right]$ is a continuous function of $E\left[\widetilde{v}_{I r}\right]$, then there exists a $\widetilde{v}_{I r}^{*}$ such that $E\left[\widetilde{v}_{I r}^{*}\right]=E\left[x^{T} \widetilde{V}^{\lambda_{I}, \widetilde{v}_{I r}^{*}} y\right]$ for $E\left[\widetilde{v}_{I r}^{*}\right] \in[E[\underline{\widetilde{r}}], E[\overline{\widetilde{r}}]]$. Furthermore, $E\left[\widetilde{v}_{I r}^{*}\right]$ is unique since $E\left[x^{T} \widetilde{V}^{\lambda_{I}, \widetilde{v}_{I r}} y\right]$ is nonincreasing on $[E[\underline{\tilde{r}}], E[\overline{\tilde{r}}]]$. Similarly, for player $J$, there exists a $\widetilde{\eta}_{J r}^{*}$ such that $E\left[\tilde{\eta}_{J r}^{*}\right]$ is unique and satisfies $E\left[\widetilde{\eta}_{I r}^{*}\right]=E\left[x^{T} \widetilde{\Gamma}^{\lambda_{J}, \tilde{\eta}_{I r}^{*}} y\right]$ for $E\left[\widetilde{\eta}_{I r}^{*}\right] \in[E[\tilde{r}], E[\overline{\tilde{r}}]]$. Thus, the best responses of player $I$ to a strategy $y^{*} \in S_{J}$ are the optimal solutions of the fuzzy expected value model

$$
\max _{x \in S_{I}} E\left[x^{T} \widetilde{V}^{\lambda_{I}, \widetilde{v}_{I r}^{*}} y^{*}\right]
$$

and the best responses of player $J$ to a strategy $x^{*} \in S_{I}$ are the optimal solutions of the fuzzy expected value model

$$
\max _{y \in S_{J}} E\left[x^{* T} \widetilde{\Gamma}^{\lambda_{J}, \tilde{\eta}_{I r}^{*}} y\right]
$$

Definition 14. A strategy profile $\left(x^{*}, y^{*}\right)$ is an expected loss aversion Nash equilibrium (ELANE) of an extended bimatrix game $G_{3}$ if $\left(x^{*}, y^{*}\right)$ satisfies

$$
\begin{aligned}
E\left[\widetilde{v}_{I r}^{*}\right]=E\left[x^{* T} \widetilde{V}^{\lambda_{I}, \widetilde{v}_{I r}^{*}} y^{*}\right] \geq E\left[x^{T} \widetilde{V}^{\lambda_{I}, \widetilde{v}_{I r}} y^{*}\right], & \\
& \forall x \in S_{I}, \\
E\left[\widetilde{\eta}_{J r}^{*}\right]=E\left[x^{* T} \widetilde{\Gamma}^{\lambda_{J}, \widetilde{\eta}_{I r}^{*}} y^{*}\right] \geq E\left[x^{* T} \widetilde{\Gamma}^{\lambda_{J}, \widetilde{\eta}_{I r}} y\right], & \\
& \forall y \in S_{J} .
\end{aligned}
$$

$\left(E\left[\widetilde{v}_{I r}^{*}\right], E\left[\widetilde{\eta}_{I r}^{*}\right]\right)$ is called an expected value of the game $G_{3}$.

Suppose that the optimistic value criterion is employed. Given an extended bimatrix game $G_{3}$ and reference points $\widetilde{v}_{I r}$ and $\tilde{\eta}_{J r}$, we have

$$
\begin{aligned}
& \widetilde{v}_{i j}^{\lambda_{I}, \widetilde{v}_{I r}} \\
& = \begin{cases}\widetilde{v}_{i j} & \text { if } \widetilde{v}_{i j \text { sup }}(\alpha) \geq \widetilde{v}_{I r \text { sup }}(\alpha) \\
\widetilde{v}_{i j}+\lambda_{I}\left(\widetilde{v}_{i j}-\widetilde{v}_{I r}\right) & \text { if } \widetilde{v}_{i j \text { sup }}(\alpha)<\widetilde{v}_{I r \text { sup }}(\alpha)\end{cases} \\
& \widetilde{\eta}_{i j}^{\lambda_{j}, \tilde{\eta}_{I r}} \\
& = \begin{cases}\widetilde{\eta}_{i j} & \text { if } \widetilde{\eta}_{i j \text { sup }}(\beta) \geq \widetilde{\eta}_{I r \text { sup }}(\beta) \\
\widetilde{\eta}_{i j}+\lambda_{J}\left(\widetilde{\eta}_{i j}-\widetilde{\eta}_{I r}\right) & \text { if } \widetilde{\eta}_{i j \text { sup }}(\beta)<\widetilde{\eta}_{I r \text { sup }}(\beta)\end{cases}
\end{aligned}
$$

where $\widetilde{v}_{i j}-\widetilde{v}_{I r}=\left(v_{i j}^{L}-v_{I r}^{L}, v_{i j}^{M}-v_{I r}^{M}, v_{i j}^{U}-v_{I r}^{U}\right), \widetilde{\eta}_{i j}-\widetilde{\eta}_{J r}=$ $\left(\eta_{i j}^{L}-\eta_{J r}^{L}, \eta_{i j}^{M}-\eta_{J r}^{M}, \eta_{i j}^{U}-\eta_{J r}^{U}\right)$.

For player $I$, it follows from Lemma 4 and Definition 9 that

$$
\begin{aligned}
& \widetilde{v}_{i j \text { sup }}(\alpha)= \begin{cases}2 \alpha v_{i j}^{M}+(1-2 \alpha) v_{i j}^{U} & \text { if } \alpha \leq 0.5 \\
(2 \alpha-1) v_{i j}^{L}+(2-2 \alpha) v_{i j}^{M} & \text { if } \alpha>0.5,\end{cases} \\
& \widetilde{v}_{I r \text { sup }}(\alpha)= \begin{cases}2 \alpha v_{I r}^{M}+(1-2 \alpha) v_{I r}^{U} & \text { if } \alpha \leq 0.5 \\
(2 \alpha-1) v_{I r}^{L}+(2-2 \alpha) v_{I r}^{M} & \text { if } \alpha>0.5\end{cases}
\end{aligned}
$$

and

$$
\begin{aligned}
\left(\widetilde{v}_{i j}-\widetilde{v}_{I r}\right)_{\text {sup }}(\alpha) & = \begin{cases}2 \alpha\left(v_{i j}^{M}-v_{I r}^{M}\right)+(1-2 \alpha)\left(v_{i j}^{U}-v_{I r}^{U}\right) & \text { if } \alpha \leq 0.5 \\
(2 \alpha-1)\left(v_{i j}^{L}-v_{I r}^{L}\right)+(2-2 \alpha)\left(v_{i j}^{M}-v_{I r}^{M}\right) & \text { if } \alpha>0.5\end{cases} \\
& = \begin{cases}\left(2 \alpha v_{i j}^{M}+(1-2 \alpha) v_{i j}^{U}\right)-\left(2 \alpha v_{I r}^{M}+(1-2 \alpha) v_{I r}^{U}\right) & \text { if } \alpha \leq 0.5 \\
\left((2 \alpha-1) v_{i j}^{L}+(2-2 \alpha) v_{i j}^{M}\right)-\left((2 \alpha-1) v_{I r}^{L}+(2-2 \alpha) v_{I r}^{M}\right) & \text { if } \alpha>0.5 .\end{cases}
\end{aligned}
$$

Thus, we have

$$
\left(\widetilde{v}_{i j}-\widetilde{v}_{I r}\right)_{\text {sup }}(\alpha)=\widetilde{v}_{i j \text { sup }}(\alpha)-\widetilde{v}_{\text {Ir sup }}(\alpha)
$$

Similarly, for player $J$, we have

$$
\left(\widetilde{\eta}_{i j}-\widetilde{\eta}_{J r}\right)_{\text {sup }}(\beta)=\widetilde{\eta}_{i j \text { sup }}(\beta)-\widetilde{\eta}_{J r \text { sup }}(\beta)
$$

For a strategy profile $(x, y)$ and a reference point pair $\left(\widetilde{v}_{I r}, \widetilde{\eta}_{I r}\right)$, players $I$ and $J$ want to maximize the optimistic value of their expected final loss aversion utility at their own confidence level. Thus, for player $I$, we have $x^{T} \widetilde{V}_{\text {sup }}^{\lambda_{I}, \widetilde{\underline{r}}}(\alpha) y=x^{T} \widetilde{V}_{\text {sup }}(\alpha) y \geq \min _{i \in M, j \in N} \widetilde{v}_{i j \text { sup }}(\alpha) \geq \underline{\underline{r}}_{\text {sup }}(\alpha)$ and $x^{T} \widetilde{V}_{\text {sup }}^{\lambda_{I}} \overline{\bar{r}}(\alpha) y \leq x^{T} \widetilde{V}_{\text {sup }}(\alpha) y \leq \max _{i \in M, j \in N} \widetilde{v}_{i j \text { sup }}(\alpha) \leq$ 
$\overline{\widetilde{r}}_{\text {sup }}(\alpha)$. Since $x^{T} \widetilde{V}_{\text {sup }}^{\lambda_{I} \widetilde{v}_{I r}}(\alpha) y$ is a continuous function of $\widetilde{v}_{I r \text { sup }}(\alpha)$, then there exists a $\widetilde{v}_{I r}^{*}$ such that $\widetilde{v}_{I r \text { sup }}^{*}(\alpha)=$ $x^{T} \widetilde{V}_{\text {sup }}^{\lambda_{I}} \widetilde{v}_{\text {Ir }}^{*}(\alpha) y$ for $\widetilde{v}_{\text {Ir sup }}^{*}(\alpha) \in\left[\underline{\underline{r}}_{\text {sup }}(\alpha), \overline{\widetilde{r}}_{\text {sup }}(\alpha)\right]$. Furthermore, $\widetilde{v}_{I r \text { sup }}^{*}(\alpha)$ is unique since $x^{T} \widetilde{V}_{\text {sup }}^{\lambda_{I}, \widetilde{v}_{I r}}(\alpha) y$ is nonincreasing on $\left[\underline{\widetilde{r}}_{\text {sup }}(\alpha), \overline{\widetilde{r}}_{\text {sup }}(\alpha)\right]$. Similarly, for player $J$, there exists a $\widetilde{\eta}_{J r}^{*}$ such that $\widetilde{\eta}_{\text {Jr sup }}^{*}(\beta)$ is unique and satisfies $\widetilde{\eta}_{\text {Jr sup }}^{*}(\beta)=x^{T} \widetilde{\Gamma}_{\text {sup }}^{\widetilde{T}_{J} \widetilde{\eta}_{j_{r}}^{*}}(\beta) y$ for $\widetilde{\eta}_{J r \text { sup }}^{*}(\beta) \in\left[\underline{\widetilde{r}}_{\text {sup }}(\beta), \overline{\widetilde{r}}_{\text {sup }}(\beta)\right]$. Thus, the best responses of player $I$ to a strategy $y^{*} \in S_{I}$ are the optimal solutions of the following chance-constrained programming model:

$$
\max _{x \in S_{I}} \max _{u_{I}} \operatorname{Cr}\left\{x^{T} \widetilde{V}^{\lambda_{I}, \widetilde{v}_{I r}} y^{*} \geq u_{I}\right\} \geq \alpha
$$

where $\alpha$ is a predetermined confidence level of player $I$ and $u_{I}$ is a real number.

And the best responses of player $J$ to a strategy $x^{*} \in S_{I}$ are the optimal solutions of the following chance-constrained programming model

$$
\max _{y \in S_{J}} \max _{u_{J}} \operatorname{Cr}\left\{x^{* T} \widetilde{\Gamma}^{\lambda_{J}, \widetilde{\eta}_{I r}} y \geq u_{J}\right\} \geq \beta
$$

where $\beta$ is a predetermined confidence level of player $J$ and $u_{J}$ is a real number.

Definition 15. A strategy profile $\left(x^{*}, y^{*}\right)$ is $(\alpha, \beta)$-optimistic loss aversion Nash equilibrium $((\alpha, \beta)$-OLANE) of an extended bimatrix game $G_{3}$ if $\left(x^{*}, y^{*}\right)$ satisfies

$$
\begin{aligned}
& \widetilde{v}_{I r \text { sup }}^{*}(\alpha)= u_{I}^{*} \\
&=\max \left\{u_{I} \mid \operatorname{Cr}\left\{x^{* T} \widetilde{V}^{\lambda_{I}, \widetilde{v}_{I r}^{*}} y^{*} \geq u_{I}\right\} \geq \alpha\right\} \\
& \geq \max \left\{u_{I} \mid \operatorname{Cr}\left\{x^{T} \widetilde{V}^{\lambda_{I}, \widetilde{v}_{I r}} y^{*} \geq u_{I}\right\} \geq \alpha\right\}, \\
& \forall x \in S_{I}
\end{aligned}
$$

and

$$
\begin{aligned}
& \widetilde{\eta}_{J r \text { sup }}^{*}(\beta)= u_{J}^{*} \\
&=\max \left\{u_{J} \mid \operatorname{Cr}\left\{x^{* T} \widetilde{\Gamma}^{\lambda_{J}, \tilde{\eta}_{I r}^{*}} y^{*} \geq u_{J}\right\} \geq \beta\right\} \\
& \geq \max \left\{u_{J} \mid \operatorname{Cr}\left\{x^{* T} \widetilde{\Gamma}^{\lambda_{J}, \widetilde{\eta}_{J r}} y \geq u_{J}\right\} \geq \beta\right\}, \\
& \forall y \in S_{J} .
\end{aligned}
$$

$\left(\widetilde{v}_{I r \text { sup }}^{*}(\alpha), \widetilde{\eta}_{I r \text { sup }}^{*}(\beta)\right)$ is called an optimistic value of the game $G_{3}$.

Suppose that the pessimistic value criterion is employed. Given an extended bimatrix game $G_{3}$ and reference points $\widetilde{v}_{I r}$ and $\widetilde{\eta}_{J r}$, we have

$$
\begin{aligned}
& \widetilde{v}_{i j}^{\lambda_{I}, \widetilde{v}_{I r}} \\
& = \begin{cases}\widetilde{v}_{i j} & \text { if } \widetilde{v}_{i j \text { inf }}(\alpha) \geq \widetilde{v}_{I r \text { inf }}(\alpha) \\
\widetilde{v}_{i j}+\lambda_{I}\left(\widetilde{v}_{i j}-\widetilde{v}_{I r}\right) & \text { if } \widetilde{v}_{i j \text { inf }}(\alpha)<\widetilde{v}_{I r \text { inf }}(\alpha)\end{cases} \\
& \widetilde{\eta}_{i j}^{\lambda_{J j}, \widetilde{\eta}_{I r}} \\
& = \begin{cases}\tilde{\eta}_{i j} & \text { if } \tilde{\eta}_{i j \text { inf }}(\beta) \geq \tilde{\eta}_{J r \text { inf }}(\beta) \\
\tilde{\eta}_{i j}+\lambda_{J}\left(\widetilde{\eta}_{i j}-\widetilde{\eta}_{J r}\right) & \text { if } \tilde{\eta}_{i j \text { inf }}(\beta)<\widetilde{\eta}_{J r \text { inf }}(\beta)\end{cases}
\end{aligned}
$$

where $\widetilde{v}_{i j}-\widetilde{v}_{I r}=\left(v_{i j}^{L}-v_{I r}^{L}, v_{i j}^{M}-v_{I r}^{M}, v_{i j}^{U}-v_{I r}^{U}\right), \widetilde{\eta}_{i j}-\widetilde{\eta}_{J r}=$ $\left(\eta_{i j}^{L}-\eta_{J r}^{L}, \eta_{i j}^{M}-\eta_{J r}^{M}, \eta_{i j}^{U}-\eta_{J r}^{U}\right)$.

For player $I$, it follows from Lemma 4 and Definition 9 that

$$
\begin{aligned}
& \widetilde{v}_{i j \text { inf }}(\alpha)= \begin{cases}(1-2 \alpha) v_{i j}^{L}+2 \alpha v_{i j}^{M} & \text { if } \alpha \leq 0.5 \\
(2-2 \alpha) v_{i j}^{M}+(2 \alpha-1) v_{i j}^{U} & \text { if } \alpha>0.5,\end{cases} \\
& \widetilde{v}_{I r \text { inf }}(\alpha)= \begin{cases}(1-2 \alpha) v_{I r}^{L}+2 \alpha v_{I r}^{M} & \text { if } \alpha \leq 0.5 \\
(2-2 \alpha) v_{I r}^{M}+(2 \alpha-1) v_{I r}^{U} & \text { if } \alpha>0.5\end{cases}
\end{aligned}
$$

and

$$
\begin{aligned}
\left(\widetilde{v}_{i j}-\widetilde{v}_{I r}\right)_{\mathrm{inf}}(\alpha) & = \begin{cases}(1-2 \alpha)\left(v_{i j}^{L}-v_{I r}^{L}\right)+2 \alpha\left(v_{i j}^{M}-v_{I r}^{M}\right) & \text { if } \alpha \leq 0.5 \\
(2-2 \alpha)\left(v_{i j}^{M}-v_{I r}^{M}\right)+(2 \alpha-1)\left(v_{i j}^{U}-v_{I r}^{U}\right) & \text { if } \alpha>0.5\end{cases} \\
& = \begin{cases}\left((1-2 \alpha) v_{i j}^{L}+2 \alpha v_{i j}^{M}\right)-\left((1-2 \alpha) v_{I r}^{L}+2 \alpha v_{I r}^{M}\right) & \text { if } \alpha \leq 0.5 \\
\left((2-2 \alpha) v_{i j}^{M}+(2 \alpha-1) v_{i j}^{U}\right)-\left((2-2 \alpha) v_{I r}^{M}+(2 \alpha-1) v_{I r}^{U}\right) & \text { if } \alpha>0.5 .\end{cases}
\end{aligned}
$$

Thus, we have

$$
\left(\widetilde{v}_{i j}-\widetilde{v}_{I r}\right)_{\text {inf }}(\alpha)=\widetilde{v}_{i j \text { inf }}(\alpha)-\widetilde{v}_{I r \text { inf }}(\alpha)
$$

Similarly, for player $J$, we have

$$
\left(\widetilde{\eta}_{i j}-\tilde{\eta}_{J r}\right)_{\mathrm{inf}}(\beta)=\tilde{\eta}_{i j \text { inf }}(\beta)-\tilde{\eta}_{J r \text { inf }}(\beta)
$$

For a strategy profile $(x, y)$ and a reference point pair $\left(\widetilde{v}_{I r}, \widetilde{\eta}_{J r}\right)$, player $I$ wants to maximize the pessimistic value of her/his expected final loss aversion utility $x^{T} \widetilde{V}^{\lambda_{I}, \widetilde{v}_{I r}} y$ at a confidence level $\alpha$ and player $J$ wants to maximize the pessimistic value of her/his expected final loss aversion utility $x^{T} \widetilde{\Gamma}^{\prime \lambda_{J}, \tilde{\eta}_{r}} y$ at a confidence level $\beta$. Thus, for player $I$, we have $x^{T} \widetilde{V}_{\text {inf }}^{\lambda, \tilde{\underline{r}}}(\alpha) y=x^{T} \widetilde{V}_{\text {inf }}(\alpha) y \geq \min _{i \in M, j \in N} \widetilde{v}_{i j \text { inf }}(\alpha) \geq \underline{\widetilde{r}}_{\text {inf }}(\alpha)$ 
and $x^{T} \widetilde{V}_{\text {inf }}^{\lambda_{I}, \overline{\bar{r}}}(\alpha) y \leq x^{T} \widetilde{V}_{\text {inf }}(\alpha) y \leq \max _{i \in M, j \in N} \widetilde{V}_{i j \text { inf }}(\alpha) \leq$ $\overline{\widetilde{r}}_{\text {inf }}(\alpha)$. Since $x^{T} \widetilde{V}_{\text {inf }}^{\lambda_{I}, \widetilde{v}_{I r}}(\alpha) y$ is a continuous function of $\widetilde{v}_{I r \text { inf }}(\alpha)$, then there exists a $\widetilde{v}_{I r}^{*}$ such that $\widetilde{v}_{I r \text { inf }}^{*}(\alpha)=$ $x^{T} \widetilde{V}_{\text {inf }}^{\lambda_{I}} \widetilde{v}_{I r}^{*}(\alpha) y$ for $\widetilde{v}_{I r \text { inf }}^{*}(\alpha) \in\left[\underline{\underline{r}}_{\text {inf }}(\alpha), \overline{\widetilde{r}}_{\text {inf }}(\alpha)\right]$. Furthermore, $\widetilde{v}_{I r \text { inf }}^{*}(\alpha)$ is unique since $x^{T} \widetilde{V}_{\text {inf }}^{\lambda_{1}, \widetilde{V}_{I r}}(\alpha) y$ is nonincreasing on $\left[\widetilde{r}_{\text {inf }}(\alpha), \overline{\widetilde{r}}_{\text {inf }}(\alpha)\right]$. Similarly, for player $J$, there exists a $\widetilde{\eta}_{J r}^{*}$ such that $\widetilde{\eta}_{J r \text { inf }}^{*}(\beta)$ is unique and satisfies $\widetilde{\eta}_{J r \text { inf }}^{*}(\beta)=x^{T} \widetilde{\Gamma}_{\text {inf }}^{\lambda_{J} \widetilde{\eta}_{I r}^{*}}(\beta) y$ for $\widetilde{\eta}_{J r \text { inf }}^{*}(\beta) \in\left[\widetilde{r}_{\text {inf }}(\beta), \overline{\widetilde{r}}_{\text {inf }}(\beta)\right]$. Thus, the best responses of player $I$ to a strategy $y^{*} \in S_{J}$ are the optimal solutions of the following chance-constrained programming model:

$$
\max _{x \in S_{I}} \max _{u_{I}} \operatorname{Cr}\left\{x^{T} \widetilde{V}^{\lambda_{I}, \widetilde{v}_{I r}} y^{*} \leq u_{I}\right\} \geq \alpha
$$

where $\alpha$ is a predetermined confidence level of player $I$ and $u_{I}$ is a real number.

The best responses of player $J$ to a strategy $x^{*} \in S_{I}$ are the optimal solutions of the following chance-constrained programming model:

$$
\max _{y \in S_{J}} \max _{u_{J}} \operatorname{Cr}\left\{x^{* T} \widetilde{\Gamma}^{\lambda_{J}, \tilde{\eta}_{I r}} y \leq u_{J}\right\} \geq \beta
$$

where $\beta$ is a predetermined confidence level of player $J$ and $u_{J}$ is a real number.

Definition 16. A strategy profile $\left(x^{*}, y^{*}\right)$ is $(\alpha, \beta)$-pessimistic loss aversion Nash equilibrium $((\alpha, \beta)$-PLANE) of an extended bimatrix game $G_{3}$ if $\left(x^{*}, y^{*}\right)$ satisfies

$$
\begin{aligned}
\widetilde{v}_{I r \text { inf }}^{*}(\alpha) & =u_{I}^{*} \\
& =\max \left\{u_{I} \mid \operatorname{Cr}\left\{x^{* T} \widetilde{V}_{\inf }^{\lambda_{I}, \widetilde{v}_{I r}^{*}} y^{*} \leq u_{I}\right\} \geq \alpha\right\} \\
& \geq \max \left\{u_{I} \mid \operatorname{Cr}\left\{x^{T} \widetilde{V}_{\inf }^{\lambda_{I}, \widetilde{v}_{I r}} y^{*} \leq u_{I}\right\} \geq \alpha\right\},
\end{aligned}
$$

$\forall x \in S_{I}$

and

$$
\begin{array}{r}
\widetilde{\eta}_{J r \text { inf }}^{*}(\beta)=u_{J}^{*} \\
=\max \left\{u_{J} \mid \operatorname{Cr}\left\{x^{* T} \widetilde{\Gamma}_{\inf }^{\lambda_{J}, \tilde{\eta}_{r r}^{*}} y^{*} \leq u_{J}\right\} \geq \beta\right\} \\
\geq \max \left\{u_{J} \mid \operatorname{Cr}\left\{x^{* T} \widetilde{\Gamma}_{\inf }^{\lambda_{J}, \tilde{\eta}_{I r}} y \leq u_{J}\right\} \geq \beta\right\}, \\
\forall y \in S_{J} .
\end{array}
$$

$\left(\widetilde{v}_{I r \text { inf }}^{*}(\alpha), \widetilde{\eta}_{J r \text { inf }}^{*}(\beta)\right)$ is called a pessimistic value of the game $G_{3}$.

3.3. Existence Theorems and Sufficient and Necessary Conditions of Credibilistic Loss Aversion Equilibria. Now, we present the existence theorems of the three credibilistic loss aversion equilibrium strategies.

Theorem 17. For any extended bimatrix game $G_{3}$, then

(1) there exists at least one $\operatorname{ELANE}\left(x^{*}, y^{*}\right)$, and the expected value of the game $G_{3}$ is $\left(x^{*} \widetilde{V}_{E}^{\lambda_{I}, \widetilde{v}_{I r}^{*}} y^{*}, x^{*} \widetilde{\Gamma}_{E}^{\lambda_{J}, \tilde{\eta}_{I r}^{*}} y^{*}\right)$, where $\widetilde{V}_{E}^{\lambda_{1}, \widetilde{v}_{I r}^{*}}=\left(E\left[\widetilde{v}_{i j}^{\lambda_{I}}, \widetilde{v}_{I r}^{*}\right]\right)_{m \times n}, \widetilde{\Gamma}_{E}^{\lambda_{J}, \widetilde{\eta}_{I r}^{*}}=\left(E\left[\widetilde{\eta}_{i j}^{\lambda_{j}, \widetilde{\eta}_{I r}^{*}}\right]\right)_{m \times n}, E\left[\widetilde{v}_{I r}^{*}\right]$ $=E\left[x^{* T} \widetilde{V}^{\lambda_{I}, \widetilde{v}_{I r}^{*}} y^{*}\right], E\left[\widetilde{\eta}_{I r}^{*}\right]=E\left[x^{* T} \widetilde{\Gamma}^{\lambda_{J}, \tilde{\eta}_{I r}^{*}} y^{*}\right]$;

(2) there exists at least one $(\alpha, \beta)$-OLANE $\left(x^{*}, y^{*}\right)$, and the $(\alpha, \beta)$-optimistic loss aversion value of the game $G_{3}$ is $\left(x^{*} \widetilde{V}_{\text {sup }}^{\lambda_{I}}{\widetilde{v_{I r}}}^{*}(\alpha) y^{*}, x^{*} \widetilde{\Gamma}_{\text {sup }}^{\lambda_{J}, \widetilde{\eta}_{I r}^{*}}(\beta) y^{*}\right)$, where $\widetilde{V}_{\text {sup }}^{\lambda_{I}, \tilde{v}_{I r}^{*}}(\alpha)=$ $\left(\widetilde{v}_{i j \text { sup }}^{\lambda_{I}, \widetilde{v}_{I r}^{*}}(\alpha)\right)_{m \times n}, \quad \widetilde{\Gamma}_{\text {sup }}^{\lambda_{J}, \tilde{\eta}_{I r}^{*}}(\beta)=\left(\widetilde{\eta}_{i j \text { sup }}^{\lambda_{J}, \tilde{\eta}_{I r}^{*}}(\beta)\right)_{m \times n}, \widetilde{v}_{I r \text { sup }}^{*}(\alpha)=$ $x^{*} \widetilde{V}_{\text {sup }}^{\lambda_{I} \widetilde{v}_{I r}^{*}}(\alpha) y^{*}, \widetilde{\eta}_{J r \text { sup }}^{*}(\beta)=x^{*} \widetilde{\Gamma}_{\text {sup }}^{\lambda_{J}, \widetilde{\eta}_{I r}^{*}}(\beta) y^{*}$;

(3) there exists at least one $(\alpha, \beta)$-PLANE $\left(x^{*}, y^{*}\right)$, and the $(\alpha, \beta)$-pessimistic loss aversion value of the game $G_{3}$ is $\left(x^{*} \widetilde{V}_{\text {inf }}^{\lambda_{I} \widetilde{V}_{I r}^{*}}(\alpha) y^{*}, x^{*} \widetilde{\Gamma}_{\text {inf }}^{\lambda_{J} \tilde{\eta}_{I r}^{*}}(\beta) y^{*}\right)$, where $\widetilde{V}_{\text {inf }}^{\lambda_{I}, \widetilde{v}_{I r}^{*}}(\alpha)=$ $\left(\widetilde{v}_{i j \text { inf }}^{\lambda_{I}, \widetilde{v}_{I r}^{*}}(\alpha)\right)_{m \times n}, \quad \widetilde{\Gamma}_{\text {inf }}^{\lambda_{J}, \tilde{\eta}_{I r}^{*}}(\beta)=\left(\widetilde{\eta}_{i j \text { inf }}^{\lambda_{I}, \widetilde{\eta}_{I r}^{*}}(\beta)\right)_{m \times n}, \widetilde{v}_{I r \text { inf }}^{*}(\alpha)=$ $x^{*} \widetilde{V}_{\text {inf }}^{\lambda_{I}, \widetilde{v}_{I r}^{*}}(\alpha) y^{*}, \widetilde{\eta}_{J r \text { inf }}^{*}(\beta)=x^{*} \widetilde{\Gamma}_{\text {inf }}^{\lambda_{J}, \widetilde{\eta}_{I r}^{*}}(\beta) y^{*}$.

Proof. The proofs of the three parts are similar. Here, for simplicity, we only prove part (2).

Given an extended game $G_{3}=\left\langle\{I, J\}, S_{I} \times S_{J}, \widetilde{V}^{\lambda_{I}}, \widetilde{\Gamma}^{\lambda_{I}}\right\rangle$, for any $\widetilde{v}_{I r}=\left(v_{I r}^{L}, v_{I r}^{M}, v_{I r}^{U}\right)$ and $\widetilde{\eta}_{J r}=\left(\eta_{J r}^{L}, \eta_{J r}^{M}, \eta_{J r}^{U}\right)$, we have $\max \left\{u_{I} \mid \operatorname{Cr}\left\{x^{T} \widetilde{V}^{\lambda_{I}, \widetilde{V}_{I r}} y \geq u_{I}\right\} \geq \alpha\right\}=$ $x^{T} \widetilde{V}_{\sup }^{\lambda_{I}, \widetilde{v}_{I r}}(\alpha) y$ and $\max \left\{u_{J} \mid \operatorname{Cr}\left\{x^{T} \widetilde{\Gamma}^{\lambda_{J}, \widetilde{\eta}_{I r}} y \geq u_{J}\right\} \geq \beta\right\}=$ $x^{T} \widetilde{\Gamma}_{\text {sup }}^{\lambda_{1}, \widetilde{\eta}_{J r}}(\beta) y$. Thus, part (2) can be proven by proving that there exists Nash equilibrium $\left(x^{*}, y^{*}\right)$ in the game $\left\langle\{I, J\}, S_{I} \times\right.$ $\left.S_{J}, \widetilde{V}_{\text {sup }}^{\lambda_{I}, \widetilde{V}_{I r}}(\alpha), \widetilde{\Gamma}_{\text {sup }}^{\lambda_{I}, \widetilde{I}_{I r}}(\beta)\right\rangle$ such that $\widetilde{v}_{I r s u p}^{*}(\alpha)=x^{*} \widetilde{V}_{\text {sup }}^{\lambda_{I}, \widetilde{V}_{I r}^{*}}(\alpha) y^{*}$, $\widetilde{\eta}_{I r \text { sup }}^{*}(\beta)=x^{*} \widetilde{\Gamma}_{\text {sup }}^{\lambda_{J}, \tilde{\eta}_{I r}^{*}}(\beta) y^{*}$. For the game $\left\langle\{I, J\}, S_{I} \times S_{J}\right.$, $\left.\widetilde{V}_{\text {sup }}^{\lambda_{I}, \tilde{V}_{I r}}(\alpha), \widetilde{\Gamma}_{\text {sup }}^{\lambda_{J}, \widetilde{\eta}_{I_{r}}}(\beta)\right\rangle$, we define the correspondence $f$ from $S_{I} \times S_{J} \times\left[\underline{\underline{r}}_{\text {sup }}(\alpha), \overline{\tilde{r}}_{\text {sup }}(\alpha)\right] \times\left[\underline{\underline{r}}_{\text {sup }}(\beta), \overline{\tilde{r}}_{\text {sup }}(\beta)\right]$ to itself as follows $\left(x^{\prime}, y^{\prime}, \widetilde{v}_{I r \text { sup }}^{\prime}(\alpha), \widetilde{\eta}_{J r \text { sup }}^{\prime}(\beta)\right) \in f\left(x, y, \widetilde{v}_{I r \text { sup }}(\alpha), \widetilde{\eta}_{J r \text { sup }}(\beta)\right)$ if $x^{\prime}\left(y^{\prime}\right)$ is a best response to $y^{\prime}\left(x^{\prime}\right)$ in the game $\left\langle\{I, J\}, S_{I} \times\right.$ $\left.S_{J}, \widetilde{V}_{\text {sup }}^{\lambda_{I}, \widetilde{V}_{I r}}(\alpha), \widetilde{\Gamma}_{\text {sup }}^{\lambda_{,}, \widetilde{\eta}_{I r}}(\beta)\right\rangle$ for player $I(J)$, where $\widetilde{v}_{I r \text { sup }}^{\prime}(\alpha)$ is the payoff that player $I$ obtains from $\left(x^{\prime}, y\right)$ and $\widetilde{\eta}_{J r \text { sup }}^{\prime}(\beta)$ is the payoff that player $J$ obtains from $\left(x, y^{\prime}\right)$ in the game $\left\langle\{I, J\}, S_{I} \times S_{J}, \widetilde{V}_{\text {sup }}^{\lambda_{I}, \widetilde{V}_{I r}}(\alpha), \widetilde{\Gamma}_{\text {sup }}^{\lambda_{J}, \widetilde{\eta}_{\text {Ir }}}(\beta)\right\rangle$.

$S_{I}, \quad S_{J}, \quad\left[\underline{\tilde{r}}_{\text {sup }}(\alpha), \overline{\tilde{r}}_{\text {sup }}(\alpha)\right] \quad$ and $\left[\underline{\tilde{r}}_{\text {sup }}(\beta), \overline{\tilde{r}}_{\text {sup }}(\beta)\right]$ are nonempty, compact, and convex, so it is their product. The correspondence is nonempty for every $\left(x, y, \widetilde{v}_{I r \text { sup }}(\alpha)\right.$, $\left.\widetilde{\eta}_{J r \text { sup }}(\beta)\right)$. For player $I(J)$, there exists a best response $x^{\prime}$ $\left(y^{\prime}\right)$ to $y(x)$ in the game $\left\langle\{I, J\}, S_{I} \times S_{J}, \widetilde{V}_{\text {sup }}^{\lambda_{I} \widetilde{v}_{I r}}(\alpha), \widetilde{\Gamma}_{\text {sup }}^{\lambda_{J}, \widetilde{\eta}_{I r}}(\beta)\right\rangle$. And let $\widetilde{v}_{\text {Irsup }}^{\prime}(\alpha)$ be the payoff that player $I$ obtains from $\left(x^{\prime}, y\right)$ and $\tilde{\eta}_{J r \text { sup }}^{\prime}(\beta)$ be the payoff that player $J$ obtains from $\left(x, y^{\prime}\right)$ in the game $\left\langle\{I, J\}, S_{I} \times S_{J}, \widetilde{V}_{\text {sup }}^{\lambda_{I}, \widetilde{v}_{I r}}(\alpha), \widetilde{\Gamma}_{\text {sup }}^{\lambda_{J}, \widetilde{\eta}_{I r}}(\beta)\right\rangle$. Then we have an element $\left(x^{\prime}, y^{\prime}, \widetilde{v}_{\text {Ir sup }}^{\prime}(\alpha), \widetilde{\eta}_{\text {Ir sup }}^{\prime}(\beta)\right)$ in $f\left(x, y, \widetilde{v}_{I r \text { sup }}(\alpha), \tilde{\eta}_{I r \text { sup }}(\beta)\right)$. If there exist at least two best responses for a player, then the payoffs that this player obtains from these best responses are the same, and so does any convex combination of these best responses. Thus, the correspondence is convex. Since functions of $\widetilde{v}_{I r \text { sup }}(\alpha)$ and $\tilde{\eta}_{\text {Jr sup }}(\beta)$ as payoffs of players $I$ and $J$ are continuous in $\left[\underline{\widetilde{r}}_{\text {sup }}(\alpha), \overline{\widetilde{r}}_{\text {sup }}(\alpha)\right]$ and $\left[\underline{\widetilde{r}}_{\text {sup }}(\beta), \overline{\widetilde{r}}_{\text {sup }}(\beta)\right]$, and the best response function has a closed graph, then the correspondence 
has a closed graph. By Kakutani's fixed point theorem, there exist $\left(x^{*}, y^{*}\right)$ and $\left(\widetilde{v}_{I r \text { sup }}^{*}(\alpha), \tilde{\eta}_{J r \text { sup }}^{*}(\beta)\right)$ such that $\left(x^{*}, y^{*}, \widetilde{v}_{I r \text { sup }}^{*}(\alpha), \widetilde{\eta}_{J r \text { sup }}^{*}(\beta)\right) \in f\left(x^{*}, y^{*}, \widetilde{v}_{I r \text { sup }}^{*}(\alpha), \widetilde{\eta}_{J r \text { sup }}^{*}(\beta)\right)$. Given payoffs of $\widetilde{v}_{I r \text { sup }}^{*}(\alpha)$ and $\widetilde{\eta}_{J r \text { sup }}^{*}(\beta)$ for players $I$ and $J$, it follows from the definition of $f$ that $\left(x^{*}, y^{*}\right)$ is a Nash equilibrium of the game $\left\langle\{I, J\}, S_{I} \times S_{J}, \widetilde{V}_{\text {sup }}^{\lambda_{I}, \widetilde{v}_{I r}^{*}}(\alpha), \widetilde{\Gamma}_{\text {sup }}^{\lambda_{J}, \widetilde{\eta}_{I r}^{*}}(\beta)\right\rangle$. Thus, there exists at least one $(\alpha, \beta)$-OLANE in the game $G_{3}$.

These complete the proof of part (2). Theorem 17 is proven.

To find the three credibilistic loss aversion Nash equilibrium strategies of the game $G_{3}$, we give the sufficient and necessary conditions.

Theorem 18. For any extended bimatrix game $G_{3}$,

(1) a strategy profile $\left(x^{*}, y^{*}\right) \in S_{I} \times S_{J}$ is an ELANE if and only if the point $\left(x^{*}, y^{*}, E\left[\widetilde{v}_{I r}^{*}\right], E\left[\widetilde{\eta}_{J r}^{*}\right]\right)$ is an optimal solution to the following quadratic programming model:

$$
\begin{array}{cl}
\max _{x, y, u_{I}, u_{J}} & x^{T}\left(\widetilde{V}_{E}^{\lambda_{I}, \widetilde{v}_{I r}}+\widetilde{\Gamma}_{E}^{\lambda_{J}, \widetilde{\eta}_{I r}}\right) y-E\left[\widetilde{v}_{I r}\right]-E\left[\widetilde{\eta}_{J r}\right] \\
\text { s.t. } & \widetilde{V}_{E}^{\lambda_{I}, \widetilde{v}_{I r}} y \leq E\left[\widetilde{v}_{I r}\right] e^{m} \\
& \widetilde{\Gamma}_{E}^{\lambda_{J}, \widetilde{\eta}_{I r}} x \leq E\left[\widetilde{\eta}_{J r}\right] e^{n} \\
& x \in S_{I}, \quad y \in S_{J} \in R
\end{array}
$$

where $\widetilde{V}_{E}^{\lambda_{I}, \widetilde{v}_{I r}}=\left(E\left[\widetilde{v}_{i j}^{\lambda_{I}, \widetilde{v}_{I r}}\right]\right)_{m \times n}, \widetilde{\Gamma}_{E}^{\lambda_{J}, \widetilde{\eta}_{I r}}=\left(E\left[\widetilde{\eta}_{i j}^{\lambda_{J}, \widetilde{\eta}_{I r}}\right]\right)_{m \times n}, E\left[\widetilde{v}_{I r}^{*}\right]$ $=E\left[x^{* T} \widetilde{V}^{\lambda_{I}, \widetilde{v}_{I r}^{*}} y^{*}\right], E\left[\widetilde{\eta}_{J r}^{*}\right]=E\left[x^{* T} \widetilde{\Gamma}^{\lambda_{J}, \widetilde{\eta}_{I r}^{*}} y^{*}\right], e^{m}$ is a $m \times 1$ vector, and $e^{n}$ is a $n \times 1$ vector.

(2) a strategy profile $\left(x^{*}, y^{*}\right) \in S_{I} \times S_{J}$ is a $(\alpha, \beta)$-OLANE if and only if the point $\left(x^{*}, y^{*}, \widetilde{v}_{\text {Ir sup }}^{*}(\alpha), \widetilde{\eta}_{J r \text { sup }}^{*}(\beta)\right)$ is an optimal solution to the following quadratic programming model:

$$
\begin{array}{cc}
\max _{x, y, u_{I}, u_{J}} & x^{T}\left(\widetilde{V}_{\text {sup }}^{\lambda_{I}, \widetilde{v}_{I r}}(\alpha)+\widetilde{\Gamma}_{\text {sup }}^{\lambda_{J}, \widetilde{\eta}_{I r}}(\beta)\right) y-\widetilde{v}_{I r \text { sup }}(\alpha) \\
& -\widetilde{\eta}_{J r \text { sup }}(\beta) \\
\text { s.t. } \quad & \widetilde{V}_{\text {sup }}^{\lambda_{I}, \widetilde{v}_{I r}}(\alpha) y \leq \widetilde{v}_{I r \text { sup }}(\alpha) e^{m} \\
& \widetilde{\Gamma}_{\text {sup }}^{\lambda_{J}, \widetilde{\eta}_{I r}}(\beta) x \leq \widetilde{\eta}_{J r \text { sup }}(\beta) e^{n} \\
& x \in S_{I}, \quad y \in S_{J}
\end{array}
$$

where $\widetilde{V}_{\text {sup }}^{\lambda_{I}, \widetilde{V}_{I r}}(\alpha)=\left(\widetilde{v}_{i j \text { sup }}^{\lambda_{I}, \widetilde{v}_{I r}}(\alpha)\right)_{m \times n}, \widetilde{\Gamma}_{\text {sup }}^{\lambda_{J}, \widetilde{\eta}_{I r}}(\beta)=\left(\widetilde{\eta}_{i j \text { sup }}^{\lambda_{J}, \widetilde{\eta}_{I r}}(\beta)\right)_{m \times n}$, $\widetilde{v}_{I r \text { sup }}^{*}(\alpha)=x^{* T} \widetilde{V}_{\text {sup }}^{\lambda_{I} \widetilde{v}_{I r}^{*}}(\alpha) y^{*}, \widetilde{\eta}_{J r \text { sup }}^{*}(\beta)=x^{* T} \widetilde{\Gamma}_{\text {sup }}^{\lambda_{J}, \widetilde{\eta}_{I r}^{*}}(\beta) y^{*}, e^{m}$ is a $m \times 1$ vector, and $e^{n}$ is a $n \times 1$ vector.

(3) a strategy profile $\left(x^{*}, y^{*}\right) \in S_{I} \times S_{J}$ is a $(\alpha, \beta)$-PLANE if and only if the point $\left(x^{*}, y^{*}, \widetilde{v}_{I r \text { inf }}^{*}(\alpha), \widetilde{\eta}_{J r \text { inf }}^{*}(\beta)\right)$ is an optimal solution to the following quadratic programming model:

$$
\begin{gathered}
\max _{x, y, u_{I}, u_{J}} x^{T}\left(\widetilde{V}_{\text {inf }}^{\lambda_{I}, \widetilde{v}_{I r}}(\alpha)+\widetilde{\Gamma}_{\text {inf }}^{\lambda_{J}, \widetilde{\eta}_{I r}}(\beta)\right) y-\widetilde{v}_{I r \text { inf }}(\alpha) \\
-\widetilde{\eta}_{J r \text { inf }}(\beta)
\end{gathered}
$$

$$
\begin{array}{ll}
\text { s.t. } & \widetilde{V}_{\inf }^{\lambda_{I}, \widetilde{v}_{I r}}(\alpha) y \leq \widetilde{v}_{I r i n f}(\alpha) e^{m} \\
& \widetilde{\Gamma}_{\inf }^{\lambda_{J}, \widetilde{\eta}_{I r}}(\beta) x \leq \widetilde{\eta}_{J r \text { inf }}(\beta) e^{n} \\
& x \in S_{I}, \quad y \in S_{J}
\end{array}
$$

where $\widetilde{V}_{\mathrm{inf}}^{\lambda_{I}, \widetilde{v}_{I r}}(\alpha)=\left(\widetilde{v}_{i j \text { inf }}^{\lambda_{I}, \widetilde{v}_{I r}}(\alpha)\right)_{m \times n}, \widetilde{\Gamma}_{\mathrm{inf}}^{\lambda_{J}, \widetilde{\eta}_{I r}}(\beta)=\left(\widetilde{\eta}_{i j \text { inf }}^{\lambda_{J}, \widetilde{\eta}_{I r}}(\beta)\right)_{m \times n}$, $\widetilde{v}_{I r \text { inf }}^{*}(\alpha)=x^{* T} \widetilde{V}_{\text {inf }}^{\lambda_{I} \widetilde{v}_{I r}^{*}}(\alpha) y^{*}, \widetilde{\eta}_{J r \text { inf }}^{*}(\beta)=x^{* T} \widetilde{\Gamma}_{\text {inf }}^{\lambda_{J}, \widetilde{\eta}_{I r}^{*}}(\beta) y^{*}, e^{m}$ is $a m \times 1$ vector, and $e^{n}$ is a $n \times 1$ vector.

Proof. The proofs of the three parts are similar. Here, for simplicity, we only prove part (2).

Firstly, assuming that a strategy profile $\left(x^{*}, y^{*}\right) \in S_{I} \times$ $S_{I}$ is an $(\alpha, \beta)$-OLANE in the game $G_{3}$, we prove that $\left(x^{*}, y^{*}, \widetilde{v}_{I r \text { sup }}^{*}(\alpha), \widetilde{\eta}_{J r \text { sup }}^{*}(\beta)\right)$ is an optimal solution of model (46).

According to Definition 15, we have

$$
\begin{aligned}
& x^{T} \widetilde{V}_{\text {sup }}^{\lambda_{I}, \widetilde{v}_{I r}}(\alpha) y^{*} \leq x^{* T} \widetilde{V}_{\text {sup }}^{\lambda_{I}, \widetilde{v}_{I r}^{*}}(\alpha) y^{*}=\widetilde{v}_{I r \text { sup }}^{*}(\alpha), \forall x \in S_{I}, \\
& x^{* T} \widetilde{\Gamma}_{\text {sup }}^{\lambda_{J}, \widetilde{\eta}_{I r}}(\beta) y \leq x^{* T} \widetilde{\Gamma}_{\text {sup }}^{\lambda_{J}, \tilde{\eta}_{I r}^{*}}(\beta) y^{*}=\widetilde{\eta}_{J r \text { sup }}^{*}(\beta), \\
& \forall y \in S_{J} .
\end{aligned}
$$

Thanks to the arbitrariness of $x$ and $y$, we have that

$$
\begin{aligned}
& \widetilde{V}_{\text {sup }}^{\lambda_{I}, \widetilde{v}_{I r}}(\alpha) y^{*} \leq \widetilde{v}_{I r \text { sup }}^{*}(\alpha) 1^{m}, \\
& \widetilde{\Gamma}_{\text {sup }}^{\lambda_{J}, \widetilde{\eta}_{I r}}(\beta) x^{*} \leq \widetilde{\eta}_{J r \text { sup }}^{*}(\beta) 1^{n},
\end{aligned}
$$

which means that the point $\left(x^{*}, y^{*}, \widetilde{v}_{I r \text { sup }}^{*}(\alpha), \widetilde{\eta}_{J r \text { sup }}^{*}(\beta)\right)$ is a feasible solution of the model (46). Obviously,

$$
\begin{gathered}
x^{T}\left(\widetilde{V}_{\text {sup }}^{\lambda_{I}, \widetilde{v}_{I r}}(\alpha)+\widetilde{\Gamma}_{\text {sup }}^{\lambda_{J}, \widetilde{\eta}_{J r}}(\beta)\right) y-\widetilde{v}_{I r \text { sup }}(\alpha) \\
-\widetilde{\eta}_{J r \text { sup }}(\beta) \leq 0, \quad \forall(x, y) \in S_{I} \times S_{J} .
\end{gathered}
$$

It follows from $x^{* T}\left(\widetilde{V}_{\text {sup }}^{\lambda_{I}, \widetilde{v}_{I r}^{*}}(\alpha)+\widetilde{\Gamma}_{\text {sup }}^{\lambda_{J}, \widetilde{\eta}_{I r}}(\beta)\right) y^{*}-\widetilde{v}_{I r \text { sup }}^{*}(\alpha)-$ $\tilde{\eta}_{J r \text { sup }}^{*}(\beta)=0$ that $\left(x^{*}, y^{*}, \widetilde{v}_{I r \text { sup }}^{*}(\alpha), \tilde{\eta}_{J r \text { sup }}^{*}(\beta)\right)$ is an optimal solution of the model (46). The necessary condition is proved.

Secondly, assume that $\left(x^{*}, y^{*}, \widetilde{v}_{I r \text { sup }}^{*}(\alpha), \widetilde{\eta}_{J r \text { sup }}^{*}(\beta)\right)$ is an optimal solution of the model (46); we prove that $\left(x^{*}, y^{*}\right) \epsilon$ $S_{I} \times S_{J}$ is an $(\alpha, \beta)$-OLANE in the game $G_{3}$.

Since the optimal solution of the model (46) is also a feasible solution of this model, we have

$$
\begin{aligned}
& \widetilde{V}_{\text {sup }}^{\lambda_{I}, \widetilde{V}_{I r}}(\alpha) y^{*} \leq \widetilde{v}_{I r \text { sup }}^{*}(\alpha) 1^{m}, \\
& \widetilde{\Gamma}_{\text {sup }}^{\lambda_{J}, \widetilde{\eta}_{I r}}(\beta) x^{*} \leq \widetilde{\eta}_{J r \text { sup }}^{*}(\beta) 1^{n} .
\end{aligned}
$$


According to the definition of the mixed strategy, we have

$$
\begin{array}{r}
x^{T} \widetilde{V}_{\text {sup }}^{\lambda_{I}, \widetilde{v}_{I r}}(\alpha) y^{*} \leq x^{* T} \widetilde{V}_{\text {sup }}^{\lambda_{I}, \widetilde{v}_{I r}^{*}}(\alpha) y^{*}=\widetilde{v}_{\text {Ir sup }}^{*}(\alpha), \\
\forall x \in S_{I}, \\
x^{* T} \widetilde{\Gamma}_{\text {sup }}^{\lambda_{J}, \widetilde{\eta}_{I r}}(\beta) y \leq x^{* T} \widetilde{\Gamma}_{\text {sup }}^{\lambda_{J}, \widetilde{\eta}_{I r}^{*}}(\beta) y^{*}=\widetilde{\eta}_{J r \text { sup }}^{*}(\beta), \\
\forall y \in S_{J} .
\end{array}
$$

This implies that the strategy profile $\left(x^{*}, y^{*}\right) \in S_{I} \times S_{I}$ is an $(\alpha, \beta)$-OLANE in the game $G_{3}$, and $\left(\widetilde{v}_{I r \text { sup }}^{*}(\alpha), \widetilde{\eta}_{J r \text { sup }}^{*}(\beta)\right)$ is the optimistic value of the game $G_{3}$. The sufficient condition is proved.

These complete the proof of part (2). Theorem 18 is proven.

3.4. Relationship of Three Credibilistic Loss Aversion Equilibria. Although we define three credibilistic loss aversion equilibria by different decision criteria, there exists some relation among the three credibilistic loss aversion equilibria.

Theorem 19. For any extended game $G_{3}=\left\langle\{I, J\}, S_{I} \times\right.$ $\left.S_{J}, \widetilde{V}^{\lambda_{I}}, \widetilde{\Gamma}^{\lambda_{J}}\right\rangle$, if $v_{i j}^{L}+v_{i j}^{U}=2 v_{i j}^{M}$ and $\eta_{i j}^{L}+\eta_{i j}^{U}=2 \eta_{i j}^{M}, a$ strategy profile $\left(x^{*}, y^{*}\right) \in S_{I} \times S_{J}$ is a credibilistic loss aversion equilibrium. Then the following propositions are equivalent:

(1) The strategy profile $\left(x^{*}, y^{*}\right) \in S_{I} \times S_{J}$ is an ELANE;

(2) The strategy profile $\left(x^{*}, y^{*}\right) \in S_{I} \times S_{J}$ is an $(0.5,0.5)$ OLANE;

(3) The strategy profile $\left(x^{*}, y^{*}\right) \in S_{I} \times S_{J}$ is a $(0.5,0.5)$ PLANE.

Proof. Since $v_{i j}^{L}+v_{i j}^{U}=2 v_{i j}^{M}, \eta_{i j}^{L}+\eta_{i j}^{U}=2 \eta_{i j}^{M}$, then it follows from Definition 7 that

$$
\begin{aligned}
& E\left[\widetilde{v}_{i j}\right]=\frac{v_{i j}^{L}+2 v_{i j}^{M}+v_{i j}^{U}}{4}=v_{i j}^{M}, \\
& E\left[\tilde{\eta}_{i j}\right]=\frac{\eta_{i j}^{L}+2 \eta_{i j}^{M}+\eta_{i j}^{U}}{4}=\eta_{i j}^{M} .
\end{aligned}
$$

From Definition 9, it follows that

$$
\begin{aligned}
\widetilde{v}_{i j s u p}(0.5) & =2 \times 0.5 v_{i j}^{M}+(1-2 \times 0.5) v_{i j}^{U}=v_{i j}^{M} \\
& =E\left[\widetilde{v}_{i j}\right] ; \\
\tilde{\eta}_{i j s u p}(0.5) & =2 \times 0.5 \eta_{i j}^{M}+(1-2 \times 0.5) \eta_{i j}^{U}=\eta_{i j}^{M} \\
& =E\left[\widetilde{\eta}_{i j}\right] .
\end{aligned}
$$

By Definition 9, we have

$$
\begin{aligned}
\widetilde{v}_{i j \text { inf }}(0.5) & =(1-2 \times 0.5) v_{i j}^{L}+2 \times 0.5 v_{i j}^{M}=v_{i j}^{M} \\
& =E\left[\widetilde{v}_{i j}\right] ; \\
\widetilde{\eta}_{i j \text { inf }}(0.5) & =(1-2 \times 0.5) \eta_{i j}^{L}+2 \times 0.5 \eta_{i j}^{M}=\eta_{i j}^{M} \\
& =E\left[\widetilde{\eta}_{i j}\right] .
\end{aligned}
$$

Thus, the games $\left\langle\{I, J\}, S_{I} \times S_{J}, E\left[\widetilde{V}^{\lambda_{I}}\right], E\left[\widetilde{\Gamma}^{\lambda_{J}}\right]\right\rangle,\left\langle\{I, J\}, S_{I} \times\right.$ $\left.S_{J}, \widetilde{V}_{\text {sup }}^{\lambda_{I}}(0.5), \widetilde{\Gamma}_{\text {sup }}^{\lambda_{J}}(0.5)\right\rangle$, and $\left\langle\{I, J\}, S_{I} \times S_{J}, \widetilde{V}_{\text {inf }}^{\lambda_{I}}(0.5), \widetilde{\Gamma}_{\text {inf }}^{\lambda_{J}}(0.5)\right\rangle$ are equivalent. By Definition 14, we have

$$
\begin{aligned}
E\left[\widetilde{v}_{I r}^{*}\right]=E\left[x^{* T} \widetilde{V}^{\lambda_{I} \widetilde{v}_{I r}^{*}} y^{*}\right] \geq E\left[x^{T} \widetilde{V}^{\lambda_{I}, \widetilde{v}_{I r}} y^{*}\right], & \\
& \forall x \in S_{I}, \\
E\left[\widetilde{\eta}_{J r}^{*}\right]=E\left[x^{* T} \widetilde{\Gamma}^{\lambda_{J}, \widetilde{\eta}_{I r}^{*}} y^{*}\right] \geq E\left[x^{* T} \widetilde{\Gamma}^{\lambda_{J}, \widetilde{\eta}_{I r}} y\right], & \\
& \forall y \in S_{J} .
\end{aligned}
$$

From Definition 15, it follows that

$$
\begin{array}{r}
\widetilde{v}_{I r \text { sup }}^{*}(0.5)=x^{* T} \widetilde{V}_{\text {sup }}^{\lambda_{I} \widetilde{v}_{I r}^{*}}(0.5) y^{*} \geq x^{T} \widetilde{V}_{\text {sup }}^{\lambda_{I}, \widetilde{v}_{I r}}(0.5) y^{*}, \\
\forall x \in S_{I}, \\
\widetilde{\eta}_{J r \text { sup }}^{*}(0.5)=x^{* T} \widetilde{\Gamma}_{\text {sup }}^{\lambda_{J}, \widetilde{\eta}_{J r}^{*}}(0.5) y^{*} \geq x^{* T} \widetilde{\Gamma}_{\text {sup }}^{\lambda_{J}, \widetilde{\eta}_{I r}}(0.5) y, \\
\forall y \in S_{J} .
\end{array}
$$

It follows from Definition 16 that

$$
\begin{array}{r}
\widetilde{v}_{\text {Ir inf }}^{*}(0.5)=x^{* T} \widetilde{V}_{\text {inf }}^{\lambda_{I} \widetilde{v}_{I r}^{*}}(0.5) y^{*} \geq x^{T} \widetilde{V}_{\text {inf }}^{\lambda_{I}, \widetilde{v}_{I r}}(0.5) y^{*}, \\
\forall x \in S_{I}, \\
\widetilde{\eta}_{J r \text { sup }}^{*}(0.5)=x^{* T} \widetilde{\Gamma}_{\text {inf }}^{\lambda_{J}, \widetilde{\tilde{I}}_{I r}^{*}}(0.5) y^{*} \geq x^{* T} \widetilde{\Gamma}_{\mathrm{inf}}^{\lambda_{J}, \widetilde{\eta}_{J r}}(0.5) y, \\
\forall y \in S_{J} .
\end{array}
$$

Thus, we have

$$
\begin{aligned}
\widetilde{v}_{I r \text { sup }}^{*}(0.5) & =E\left[x^{* T} \widetilde{V}^{\lambda_{I}, \widetilde{v}_{I r}^{*}} y^{*}\right]=E\left[\widetilde{v}_{I r}^{*}\right] \\
& \geq E\left[x^{T} \widetilde{V}^{\lambda_{I}, \widetilde{v}_{I r}} y^{*}\right], \quad \forall x \in S_{I}, \\
\widetilde{\eta}_{J r \text { sup }}^{*}(0.5) & =E\left[x^{* T} \widetilde{\Gamma}^{\lambda_{J}, \widetilde{\eta}_{I r}^{*}} y^{*}\right]=E\left[\widetilde{\eta}_{J r}^{*}\right] \\
& \geq E\left[x^{* T} \widetilde{\Gamma}^{\lambda_{J}, \widetilde{\eta}_{I r}} y\right], \quad \forall y \in S_{J} ; \\
\widetilde{v}_{I r \text { inf }}^{*}(0.5) & =E\left[x^{* T} \widetilde{V}^{\lambda_{I}, \widetilde{v}_{I r}^{*}} y^{*}\right]=E\left[\widetilde{v}_{I r}^{*}\right] \\
& \geq E\left[x^{T} \widetilde{V}^{\lambda_{I}, \widetilde{v}_{I r}} y^{*}\right], \quad \forall x \in S_{I}, \\
\widetilde{\eta}_{J r \text { sup }}^{*}(0.5) & =E\left[x^{* T} \widetilde{\Gamma}^{\lambda_{J}, \widetilde{\eta}_{I r}^{*}} y^{*}\right]=E\left[\widetilde{\eta}_{J r}^{*}\right] \\
& \geq E\left[x^{* T} \widetilde{\Gamma}^{\lambda_{J}, \widetilde{\eta}_{I r}} y\right], \quad \forall y \in S_{J} .
\end{aligned}
$$

Thus, $\left(x^{*}, y^{*}\right) \in S_{I} \times S_{J}$ is an ELANE, $(0.5,0.5)$ OLANE and $(0.5,0.5)$-PLANE of the extended game $G_{3}$. These complete the proof of Theorem 19.

\section{Analysis of Credibilistic Loss Aversion Nash Equilibria}

In this section, we discuss the relation between credibilistic loss aversion equilibria and loss aversion coefficients in a $2 \times 2$ bimatrix game. 
To discuss the relation between credibilistic loss aversion equilibria and loss aversion coefficients, we consider a game $G_{2}=\left\langle\{I, J\}, S_{I} \times S_{J}, \widetilde{V}, \widetilde{\Gamma}\right\rangle$, where the payoff matrices of players $I$ and $J$ are given, respectively,

$$
\begin{aligned}
\widetilde{V} & =\left[\begin{array}{ll}
(2,4,8) & (1,2,3) \\
(1,2,3) & (1,3,7)
\end{array}\right], \\
\widetilde{\Gamma} & =\left[\begin{array}{ll}
(1,3,7) & (1,2,3) \\
(1,2,3) & (2,4,8)
\end{array}\right] .
\end{aligned}
$$

An extended bimatrix game with triangular fuzzy payoffs $G_{3}=\left\langle\{I, J\}, S_{I} \times S_{J}, \widetilde{V}^{\lambda_{I}}, \widetilde{\Gamma}^{\lambda_{J}}\right\rangle$ has two additional elements-the loss aversion coefficients of players $I$ and $J$. $\lambda_{I}, \lambda_{I} \in R_{+}$specify the levels of loss aversion of players $I$ and $J$, respectively. Let $\left(x_{1}, 1-x_{1}\right)$ and $\left(y_{1}, 1-y_{1}\right)$ be strategies played by players $I$ and $J$, respectively. And let $\widetilde{v}_{I r}=\left(v_{I r}^{L}, v_{I r}^{M}, v_{I r}^{U}\right)$ and $\tilde{\eta}_{J r}=\left(\eta_{J r}^{L}, \eta_{J r}^{M}, \eta_{J r}^{U}\right)$ be reference points of players $I$ and $J$.

4.1. Credibilistic Loss Aversion Nash Equilibria and Loss Aversion Coefficients. Here, we analyze the impact of loss aversion coefficients on three credibilistic loss aversion equilibria.

When players employ the expected value criterion, then we have

$$
\begin{aligned}
& E[\widetilde{V}]=\left[\begin{array}{cc}
4.5 & 2 \\
2 & 3.5
\end{array}\right], \\
& E[\widetilde{\Gamma}]=\left[\begin{array}{cc}
3.5 & 2 \\
2 & 4.5
\end{array}\right] .
\end{aligned}
$$

According to part (1) of Theorem 18, the ELANE of the extended bimatrix game $G_{3}$ satisfies

$$
\begin{aligned}
& 4.5 y_{1}^{*}+\left(2-\lambda_{I}\left(E\left[\widetilde{v}_{I r}^{*}\right]-2\right)\right)\left(1-y_{1}^{*}\right) \\
& \quad=\left(2-\lambda_{I}\left(E\left[\widetilde{v}_{I r}^{*}\right]-2\right)\right) y_{1}^{*}+3.5\left(1-y_{1}^{*}\right) \\
& \quad=E\left[\widetilde{v}_{I r}^{*}\right]
\end{aligned}
$$

and

$$
\begin{aligned}
& 3.5 x_{1}^{*}+\left(2-\lambda_{J}\left(E\left[\widetilde{\eta}_{J r}^{*}\right]-2\right)\right)\left(1-x_{1}^{*}\right) \\
& \quad=\left(2-\lambda_{J}\left(E\left[\widetilde{\eta}_{J r}^{*}\right]-2\right)\right) x_{1}^{*}+4.5\left(1-x_{1}^{*}\right) \\
& \quad=E\left[\widetilde{\eta}_{J r}^{*}\right],
\end{aligned}
$$

where $\widetilde{v}_{I r}^{*}$ and $\widetilde{\eta}_{J r}^{*}$ are the reference points of players $I$ and $J$, respectively.
Thus, we have

$$
\begin{aligned}
& x_{1}^{*}=1-\frac{-3 \lambda_{J}-4+\sqrt{16+15 \lambda_{J}\left(\lambda_{J}+2\right)}}{2 \lambda_{J}}, \\
& y_{1}^{*}=\frac{-3 \lambda_{I}-4+\sqrt{16+15 \lambda_{I}\left(\lambda_{I}+2\right)}}{2 \lambda_{I}}, \\
& E\left[\widetilde{v}_{I r}^{*}\right] \\
& =\frac{0.5 \lambda_{I}+2\left(\lambda_{I}-1\right)\left[4\left(1+\lambda_{I}\right)-\sqrt{16+15 \lambda_{I}\left(\lambda_{I}+2\right)}\right]}{\lambda_{I}\left(4\left(1+\lambda_{I}\right)-\sqrt{16+15 \lambda_{I}\left(\lambda_{I}+2\right)}\right)}, \\
& E\left[\widetilde{\eta}_{J r}^{*}\right] \\
& =\frac{0.5 \lambda_{J}+2\left(\lambda_{J}-1\right)\left[4\left(1+\lambda_{J}\right)-\sqrt{16+15 \lambda_{J}\left(\lambda_{J}+2\right)}\right]}{\lambda_{J}\left(4\left(1+\lambda_{J}\right)-\sqrt{16+15 \lambda_{J}\left(\lambda_{J}+2\right)}\right)} .
\end{aligned}
$$

When players employ the optimistic value criterion, to discuss the impact of loss aversion coefficients on $(\alpha, \beta)$ OLANE, let $\alpha=0.8, \beta=0.7$. Then we have

$$
\begin{aligned}
& \widetilde{V}_{\text {sup }}(0.8)=\left[\begin{array}{ll}
2.8 & 1.4 \\
1.4 & 1.8
\end{array}\right], \\
& \widetilde{\Gamma}_{\text {sup }}(0.7)=\left[\begin{array}{ll}
2.2 & 1.6 \\
1.6 & 3.2
\end{array}\right] .
\end{aligned}
$$

According to part (2) of Theorem 18, the $(0.8,0.7)$ OLANE of the extended bimatrix game $G_{3}$ satisfies

$$
\begin{aligned}
& 2.8 y_{1}^{*}+\left(1.4-\lambda_{I}\left(\widetilde{v}_{I r \text { sup }}^{*}(0.8)-1.4\right)\right)\left(1-y_{1}^{*}\right) \\
& \quad=\left(1.4-\lambda_{I}\left(\widetilde{v}_{I r \text { sup }}^{*}(0.8)-1.4\right)\right) y_{1}^{*}+1.8\left(1-y_{1}^{*}\right) \\
& \quad=\widetilde{v}_{I r \text { sup }}^{*}(0.8)
\end{aligned}
$$

and

$$
\begin{aligned}
2.2 x_{1}^{*}+\left(1.6-\lambda_{J}\left(\widetilde{\eta}_{J r \text { sup }}^{*}(0.7)-1.6\right)\right)\left(1-x_{1}^{*}\right) \\
\quad=\left(1.6-\lambda_{J}\left(\widetilde{\eta}_{J r \text { sup }}^{*}(0.7)-1.6\right)\right) x_{1}^{*}+3.2\left(1-x_{1}^{*}\right) \\
=\widetilde{\eta}_{J r \text { sup }}^{*}(0.7),
\end{aligned}
$$

where $\widetilde{v}_{I r}^{*}$ and $\widetilde{\eta}_{I r}^{*}$ are the reference points of players $I$ and $J$, respectively.

Thus, we have

$$
\begin{aligned}
& x_{1}^{*}=1-\frac{-6 \lambda_{J}-11+\sqrt{121+96 \lambda_{J}\left(\lambda_{J}+2\right)}}{10 \lambda_{J}}, \\
& y_{1}^{*}=\frac{-4 \lambda_{I}-9+\sqrt{81+56 \lambda_{I}\left(\lambda_{I}+2\right)}}{10 \lambda_{I}},
\end{aligned}
$$




$$
\begin{aligned}
& \widetilde{v}_{I r \text { sup }}^{*}(0.8)=\frac{\left(0.04-0.14 \lambda_{I}\right) \sqrt{81+56 \lambda_{I}\left(\lambda_{I}+2\right)}+0.7 \lambda_{I}\left(0.8 \lambda_{I}-1\right)-0.36}{\lambda_{I}\left(0.4 \lambda_{I}+0.1-0.1 \sqrt{81+56 \lambda_{I}\left(\lambda_{I}+2\right)}\right)}, \\
& \widetilde{\eta}_{I r \text { sup }}^{*}(0.7)=\frac{\left(0.06-0.16 \lambda_{J}\right) \sqrt{121+96 \lambda_{J}\left(\lambda_{J}+2\right)}+0.96 \lambda_{J}^{2}-0.8 \lambda_{J}-0.66}{\lambda_{J}\left(0.6 \lambda_{J}+0.1-0.1 \sqrt{121+96 \lambda_{J}\left(\lambda_{J}+2\right)}\right)} .
\end{aligned}
$$

When players employ the pessimistic value criterion, to discuss the impact of loss aversion coefficients on $(\alpha, \beta)$ PLANE, let $\alpha=0.8, \beta=0.7$. Then we have

$$
\begin{aligned}
& \widetilde{V}_{\text {inf }}(0.8)=\left[\begin{array}{ll}
6.4 & 2.6 \\
2.6 & 5.4
\end{array}\right], \\
& \widetilde{\Gamma}_{\text {inf }}(0.7)=\left[\begin{array}{ll}
4.6 & 2.4 \\
2.4 & 5.6
\end{array}\right] .
\end{aligned}
$$

According to part (3) of Theorem 18, the $(0.8,0.7)$ PLANE of the extended bimatrix game $G_{3}$ satisfies

$$
6.4 y_{1}^{*}+\left(2.6-\lambda_{I}\left(\widetilde{v}_{I r \text { inf }}^{*}(0.8)-2.6\right)\right)\left(1-y_{1}^{*}\right)
$$

$$
\begin{aligned}
& =\left(2.6-\lambda_{I}\left(\widetilde{v}_{I r \text { inf }}^{*}(0.8)-2.6\right)\right) y_{1}^{*}+5.4\left(1-y_{1}^{*}\right) \\
& =\widetilde{v}_{I r \text { inf }}^{*}(0.8), \\
4.6 & x_{1}^{*}+\left(2.4-\lambda_{J}\left(\widetilde{\eta}_{J r \text { inf }}^{*}(0.7)-2.4\right)\right)\left(1-x_{1}^{*}\right) \\
= & \left(2.4-\lambda_{J}\left(\widetilde{\eta}_{J r \text { inf }}^{*}(0.7)-2.4\right)\right) x_{1}^{*}+5.6\left(1-x_{1}^{*}\right) \\
= & \widetilde{\eta}_{J r \text { inf }}^{*}(0.7),
\end{aligned}
$$

where $\widetilde{v}_{I r}^{*}$ and $\widetilde{\eta}_{J r}^{*}$ are the reference points of players $I$ and $J$, respectively.

Thus, we have

$$
\begin{aligned}
x_{1}^{*} & =1-\frac{-22 \lambda_{J}-27+\sqrt{729+704 \lambda_{J}\left(\lambda_{J}+2\right)}}{10 \lambda_{J}}, \\
y_{1}^{*} & =\frac{-28 \lambda_{I}-33+\sqrt{1089+1064 \lambda_{I}\left(\lambda_{I}+2\right)}}{10 \lambda_{I}}, \\
\widetilde{v}_{I r \text { inf }}^{*}(0.8) & =\frac{\left(0.66-0.52 \lambda_{I}\right) \sqrt{1089+1064 \lambda_{I}\left(\lambda_{I}+2\right)}+\lambda_{I}\left(17.16 \lambda_{I}-4.12\right)-21.78}{\lambda_{I}\left(6.6\left(1+\lambda_{I}\right)-0.2 \sqrt{1089+1064 \lambda_{I}\left(\lambda_{I}+2\right)}\right)}, \\
\widetilde{\eta}_{I r \text { inf }}^{*}(0.7) & =\frac{\left(0.54-0.48 \lambda_{J}\right) \sqrt{729+704 \lambda_{J}\left(\lambda_{J}+2\right)}+\lambda_{J}\left(12.96 \lambda_{J}-1.12\right)-14.58}{\lambda_{J}\left(5.4\left(1+\lambda_{J}\right)-0.2 \sqrt{729+704 \lambda_{J}\left(\lambda_{J}+2\right)}\right)} .
\end{aligned}
$$

The impact of loss aversion coefficients on three credibilistic loss aversion Nash equilibria is shown in Figures 1 and 2. The impact of loss aversion coefficients on equilibrium utilities is shown in Figures 3 and 4.

In Figure $1, y_{1}^{*}$ is increasing as $\lambda_{I}$. In Figure $2, x_{1}^{*}$ is decreasing as $\lambda_{J}$. In Figures 3 and 4 , the equilibrium utilities of player $i(i=I, J)$ are decreasing as $\lambda_{i}$. This means that a more loss-averse player has a higher probability of receiving his/her preferred outcome in equilibrium, but receives a lower utility. From Figure 1, the probability that player $I$ receives his/her preferred outcome is the highest in the three credibilistic loss aversion Nash equilibria when players employ the pessimistic value criterion, followed by the probability when players employ the expected value criterion, while it is the lowest when players employ the optimistic value criterion. A similar result for the probability that player $J$ receives his/her preferred outcome can be obtained in Figure 2. From Figure 3, the equilibrium utility of player $I$ is the highest when players employ the pessimistic value criterion, while it is the lowest when players employ the optimistic value criterion. We declare a similar result in Figure 4. From Figures 3 and 4, player is loss aversion coefficient has no impact on player $j$ 's equilibrium utility, where $i, j=I$, $J$, and $i \neq j$. 


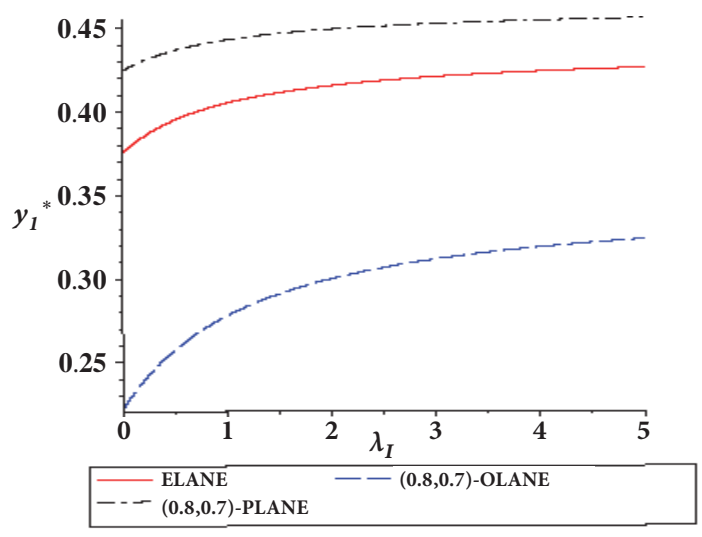

Figure 1: The impact of $\lambda_{I}$ on the credibilistic loss aversion equilibria.

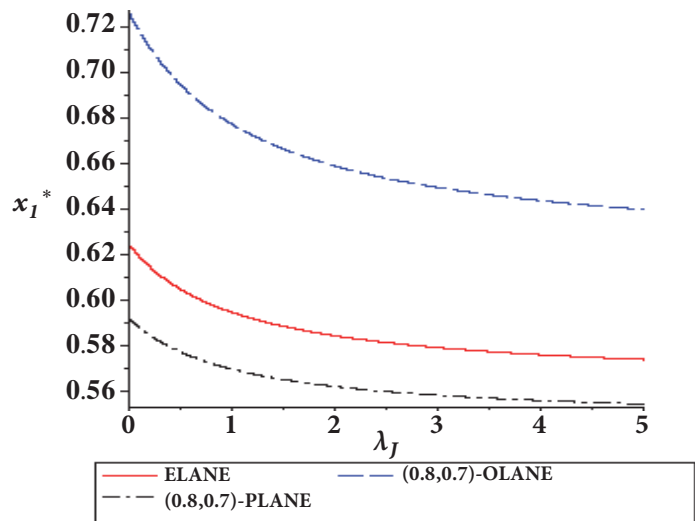

Figure 2: The impact of $\lambda_{J}$ on the credibilistic loss aversion equilibria.

4.2. Credibilistic Loss Aversion Nash Equilibria and Confidence Levels. Now, we focus on the impact of confidence levels $\alpha$ and $\beta$ on three credibilistic loss aversion equilibria. When players employ the optimistic value criterion, we distinguish four cases, i.e., $\alpha \leq 0.5, \beta \leq 0.5 ; \alpha \leq 0.5, \beta>0.5 ; \alpha>0.5, \beta \leq$ 0.5 ; and $\alpha>0.5, \beta>0.5$. For each case, let $\lambda_{I}=1$ and $\lambda_{J}=2$. For simplicity, we restrict ourselves to the case where $\alpha \leq 0.5$, $\beta>0.5$, since this consists of all possible values of confidence levels. Then we have

$$
\begin{aligned}
& \widetilde{V}_{\text {sup }}(\alpha)=\left[\begin{array}{cc}
8(1-\alpha) & 3-2 \alpha \\
3-2 \alpha & 7-8 \alpha
\end{array}\right], \\
& \widetilde{\Gamma}_{\text {sup }}(\beta)=\left[\begin{array}{ll}
5-4 \beta & 3-2 \beta \\
3-2 \beta & 6-4 \beta
\end{array}\right] .
\end{aligned}
$$

According to part (2) of Theorem 18, the $(\alpha, \beta)$-OLANE of the extended bimatrix game $G_{3}$ satisfies

$$
\begin{aligned}
& 8(1-\alpha) y_{1}^{*}+\left[2(3-2 \alpha)-\widetilde{v}_{I r \text { sup }}^{*}(\alpha)\right]\left(1-y_{1}^{*}\right) \\
& \quad=\left[2(3-2 \alpha)-\widetilde{v}_{I r \text { sup }}^{*}(\alpha)\right] y_{1}^{*}+(7-8 \alpha)\left(1-y_{1}^{*}\right) \\
& \quad=\widetilde{v}_{I r \text { sup }}^{*}(\alpha)
\end{aligned}
$$

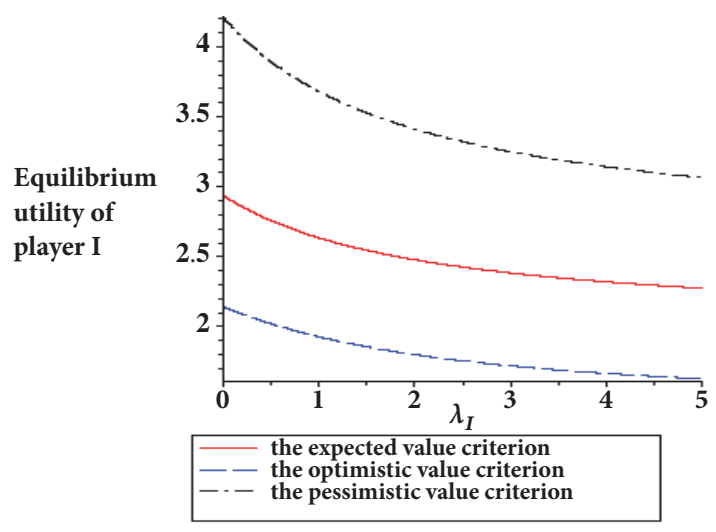

FIgURE 3: The impact of $\lambda_{I}$ on the equilibrium utilities of player $I$.

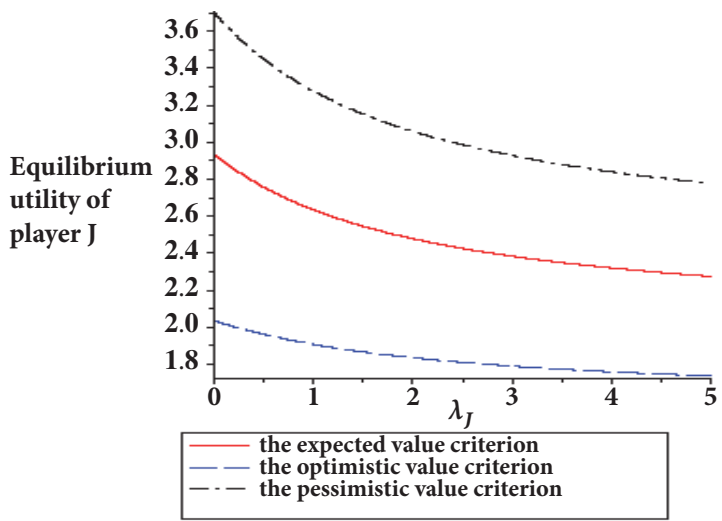

FIGURE 4: The impact of $\lambda_{J}$ on the equilibrium utilities of player $J$.

and

$$
\begin{aligned}
& (5-4 \beta) x_{1}^{*}+\left[3(3-2 \beta)-2 \widetilde{\eta}_{J r \text { sup }}^{*}(\beta)\right]\left(1-x_{1}^{*}\right) \\
& =\left[3(3-2 \beta)-2 \widetilde{\eta}_{J r \text { sup }}^{*}(\beta)\right] x_{1}^{*} \\
& \quad+(6-4 \beta)\left(1-x_{1}^{*}\right)=\widetilde{\eta}_{J r \text { sup }}^{*}(\beta),
\end{aligned}
$$

where $\widetilde{v}_{I r}^{*}$ and $\widetilde{\eta}_{J r}^{*}$ are the reference points of players $I$ and $J$, respectively.

Thus, we have

$$
\begin{aligned}
& x_{1}^{*}=-3 \beta+4.25-0.25 \sqrt{144 \beta^{2}-360 \beta+217}, \\
& y_{1}^{*}=12 \alpha-8.5+0.5 \sqrt{576 \alpha^{2}-864 \alpha+321}, \\
& \widetilde{v}_{\text {Ir sup }}^{*}(\alpha) \\
& =\frac{(4 \alpha-2) \sqrt{576 \alpha^{2}-864 \alpha+321}+96 \alpha^{2}-108 \alpha+22}{24 \alpha-21+\sqrt{576 \alpha^{2}-864 \alpha+321}}, \\
& \widetilde{\eta}_{I r \text { sup }}^{*}(\beta) \\
& =\frac{(7-4 \beta) \sqrt{144 \beta^{2}-360 \beta+217}-48 \beta^{2}+144 \beta-107}{48 \beta-60+4 \sqrt{144 \beta^{2}-360 \beta+217}} .
\end{aligned}
$$


When players employ the pessimistic value criterion, we distinguish four cases, i.e., $\alpha \leq 0.5, \beta \leq 0.5 ; \alpha \leq 0.5, \beta>0.5$; $\alpha>0.5, \beta \leq 0.5$; and $\alpha>0.5, \beta>0.5$. For each case, let $\lambda_{I}=$ 1 and $\lambda_{J}=2$. We also restrict ourselves to the case where $\alpha \leq$ $0.5, \beta>0.5$. Then we have

$$
\begin{aligned}
& \widetilde{V}_{\text {inf }}(\alpha)=\left[\begin{array}{ll}
2+4 \alpha & 1+2 \alpha \\
1+2 \alpha & 1+4 \alpha
\end{array}\right], \\
& \widetilde{\Gamma}_{\text {inf }}(\beta)=\left[\begin{array}{cc}
8 \beta-1 & 1+2 \beta \\
1+2 \beta & 8 \beta
\end{array}\right] .
\end{aligned}
$$

According to part (3) of Theorem 18, the $(\alpha, \beta)$-PLANE of the extended bimatrix game $G_{3}$ satisfies

$$
\begin{aligned}
(2 & +4 \alpha) y_{1}^{*}+\left[2(1+2 \alpha)-\widetilde{v}_{I r \text { sup }}^{*}(\alpha)\right]\left(1-y_{1}^{*}\right) \\
& =\left[2(1+2 \alpha)-\widetilde{v}_{I r \text { sup }}^{*}(\alpha)\right] y_{1}^{*}+(1+4 \alpha)\left(1-y_{1}^{*}\right) \\
& =\widetilde{v}_{I r \text { sup }}^{*}(\alpha)
\end{aligned}
$$

and

$$
\begin{aligned}
& (8 \beta+1) x_{1}^{*}+\left[3(1+2 \beta)-2 \widetilde{\eta}_{I r \text { sup }}^{*}(\beta)\right]\left(1-x_{1}^{*}\right) \\
& \quad=\left[3(1+2 \beta)-2 \widetilde{\eta}_{J r \text { sup }}^{*}(\beta)\right] x_{1}^{*}+8 \beta\left(1-x_{1}^{*}\right) \\
& \quad=\widetilde{\eta}_{J r \text { sup }}^{*}(\beta),
\end{aligned}
$$

where $\widetilde{v}_{I r}^{*}$ and $\widetilde{\eta}_{J r}^{*}$ are the reference points of players $I$ and $J$, respectively.

Thus, we have

$$
\begin{aligned}
& x_{1}^{*}=-9 \beta+1.25+0.25 \sqrt{1296 \beta^{2}-216 \beta+1}, \\
& y_{1}^{*}=-4 \alpha-0.5+0.5 \sqrt{1+32 \alpha(1+2 \alpha)}, \\
& \widetilde{v}_{I r \text { inf }}^{*}(\alpha)=\frac{-1.5-4 \alpha+0.5 \sqrt{1+32 \alpha(1+2 \alpha)}}{-8 \alpha-2+\sqrt{1+32 \alpha(1+2 \alpha)}}, \\
& \widetilde{\eta}_{I r \text { inf }}^{*}(\beta) \\
& =\frac{(\beta-1) \sqrt{1296 \beta^{2}-216 \beta+1}-36 \beta^{2}+53 \beta+1}{1+36 \beta+\sqrt{1296 \beta^{2}-216 \beta+1}} .
\end{aligned}
$$

The impact of confidence levels on three credibilistic loss aversion Nash equilibria is shown in Figures 5 and 6 . The impact of confidence levels on equilibrium utilities is shown in Figures 7 and 8.

In Figure $5, y_{1}^{*}$ is increasing (decreasing) as $\alpha$ when players employ the pessimistic (optimistic) value criterion. In Figure $6, x_{1}^{*}$ is increasing as $\beta$ when players employ the pessimistic and optimistic value criteria. In Figure 5, the probability of receiving preferred outcome for player $I$ when the optimistic value criterion is employed is higher than that when the pessimistic value criterion is employed. The probability of receiving preferred outcome for player $I$ when the pessimistic value criterion is employed is lower

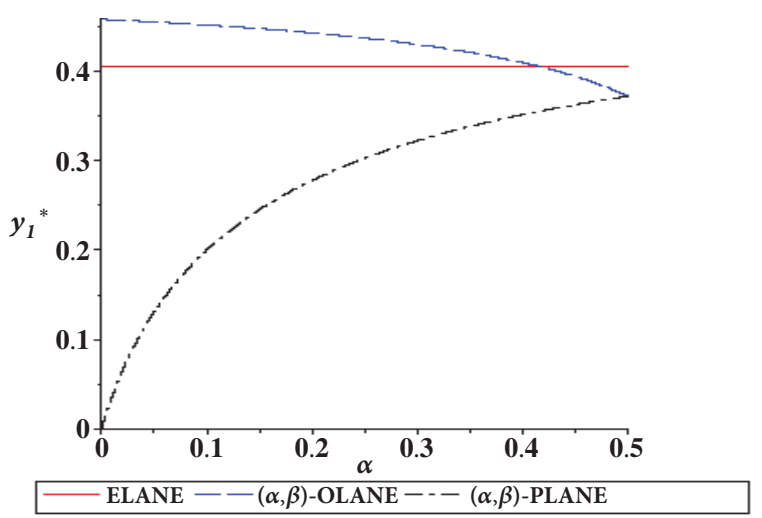

FIgURE 5: The impact of $\alpha$ on the credibilistic loss aversion equilibria.

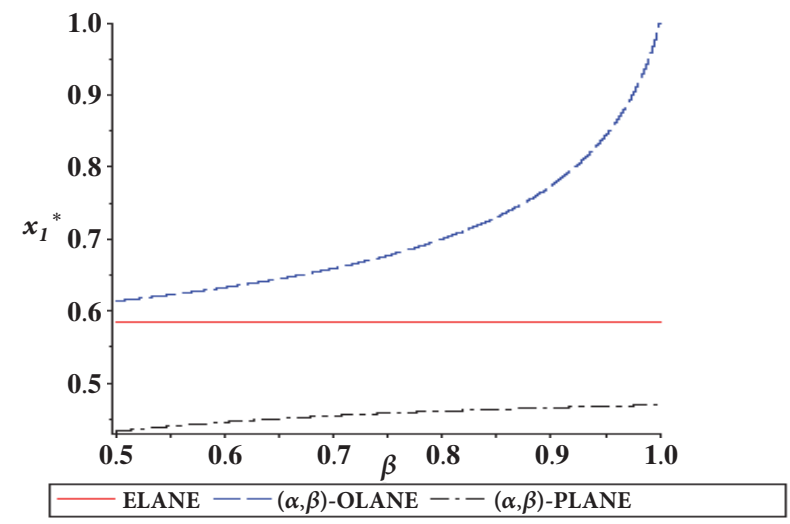

FIgURE 6: The impact of $\beta$ on the credibilistic loss aversion equilibria.

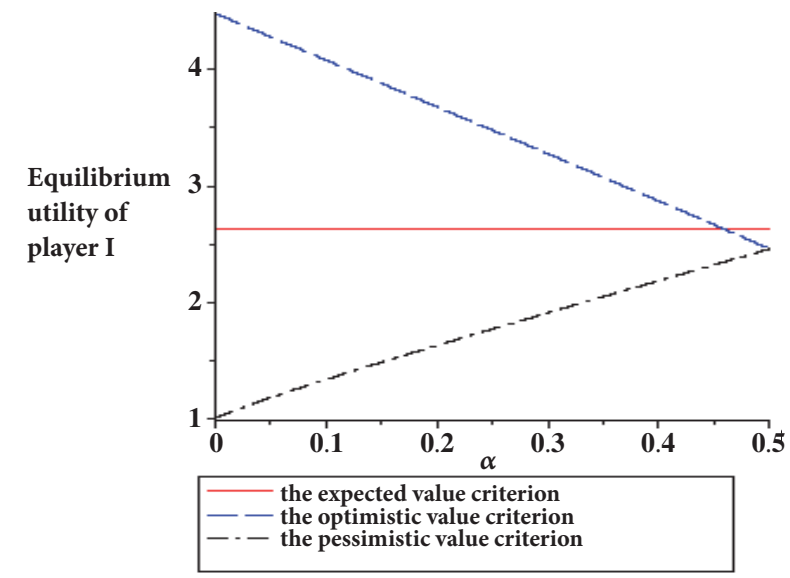

Figure 7: The impact of $\alpha$ on the equilibrium utilities of player $I$.

than that when the expected value criterion is employed, while there exists a threshold for a player's confidence levels such that the probability of receiving preferred outcome for player $I$ when the optimistic value criterion is employed is higher (lower) than that when the expected value criterion is employed if his confidence level is lower (higher) than 


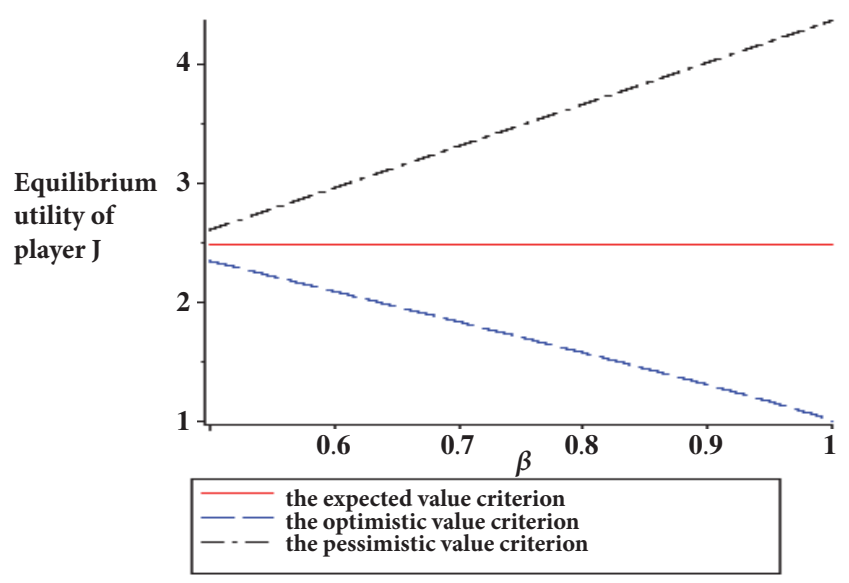

FIgURE 8: The impact of $\beta$ on the equilibrium utilities of player $J$.

this threshold. From Figure 6, the probability that player $J$ receives his/her preferred outcome is the highest in the three credibilistic loss aversion Nash equilibria when players employ the pessimistic value criterion, followed by the probability when players employ the expected value criterion, while it is the lowest when players employ the optimistic value criterion.

In Figures 7 and 8, the equilibrium utilities of players are decreasing (increasing) as their own confidence levels when players employ the optimistic (pessimistic) value criterion. When a player's confidence levels are low (i.e., $\leq 0.5$ ), from Figure 7, the equilibrium utilities of this player when players employ the pessimistic value criterion are lower than those when players employ the expected value criterion. On the other hand, when players employ the optimistic value criterion, there exists a threshold for this player's confidence levels such that the equilibrium utilities of this player are higher (lower) than those when players employ the expected value criterion if the confidence level is lower (higher) than this threshold. If a player's confidence levels are high (i.e., $>0.5$ ), from Figure 8, the equilibrium utility of this player when players employ the pessimistic value criterion is the highest in the three equilibrium utilities, followed by that when players employ the expected value criterion; it is the lowest when players employ the optimistic value criterion.

\section{Conclusion}

In this paper, considering the situation where players are loss-averse under a fuzzy decision-making environment, we investigate the effect of loss aversion on bimatrix games with triangular fuzzy payoffs. In order to describe loss aversion of players under a fuzzy decision-making environment, a loss aversion utility function with triangular fuzzy parameters is proposed. We define the solution concepts of three credibilistic loss aversion equilibria and present their existence theorems. In order to find the three credibilistic loss aversion equilibria, we present the sufficient and necessary conditions.
Moreover, the relationship among three credibilistic loss aversion equilibria is discussed. Finally, we investigate the impact of loss aversion coefficients and confidence levels on the three credibilistic loss aversion Nash equilibria for $2 \times 2$ bimatrix game with triangular fuzzy payoffs. It is found that an increase of loss aversion levels of a player leads to a decrease of his/her own payoff. We also find that the equilibrium utilities of players are decreasing (increasing) as their own confidence levels when players employ the optimistic (pessimistic) value criterion. This paper investigates the effect of loss aversion on bimatrix game with triangular fuzzy payoffs. It is interesting to extend this study to $n$-person noncooperative games, where $n>2$.

\section{Data Availability}

The data used to support the findings of this study are included within the article.

\section{Conflicts of Interest}

The authors declare that there are no conflicts of interest regarding the publication of this paper.

\section{Acknowledgments}

This work was partly supported by National Natural Science Foundation of China (71671188), Beijing Intelligent Logistics System Collaborative Innovation Center (BILSCIC-2018KF04), and Natural Science Foundation of Hunan Province, China (2016JJ1024).

\section{References}

[1] J. Nash, "Equilibrium points in $N$-person games," Proceedings of the National Acadamy of Sciences of the United States of America, vol. 36, pp. 48-49, 1950.

[2] J. C. Harsanyi, "Games with incomplete information played by "Bayesian" players. I. The basic model," Management Science, vol. 14, pp. 159-182, 1967.

[3] J. Berg, "Statistical mechanics of random two-player games," Physical Review E: Statistical, Nonlinear, and Soft Matter Physics, vol. 61, no. 3, pp. 2327-2339, 2000.

[4] R. A. Blau, "Random-payoff two-person zero-sum games," Operations Research, vol. 22, no. 6, pp. 1243-1251, 1974.

[5] R. G. Cassidy, C. A. Field, and M. J. L. Kirby, "Solution of a satisficing model for random payoff games," Management Science, vol. 19, no. 3, pp. 266-271, 1972.

[6] A. Charnes, M. J. Kirby, and W. M. Raike, "Zero-zero chanceconstrained games," Theory of Probability \& Its Applications, vol. 13, no. 4, pp. 628-646, 1968.

[7] L. Ein-Dor and I. Kanter, "Matrix games with nonuniform payoff distributions," Physica A: Statistical Mechanics and its Applications, vol. 302, no. 1-4, pp. 80-88, 2001.

[8] D. P. Roberts, "Nash equilibria of Cauchy-random zero-sum and coordination matrix games," International Journal of Game Theory, vol. 34, no. 2, pp. 167-184, 2006.

[9] L. Campos, "Fuzzy linear programming models to solve fuzzy matrix games," Fuzzy Sets and Systems, vol. 32, no. 3, pp. 275289, 1989. 
[10] A. C. Cevikel and M. Ahlatçioglu, "Solutions for fuzzy matrix games," Computers \& Mathematics with Applications, vol. 60, no. 3, pp. 399-410, 2010.

[11] S. Chandra and A. Aggarwal, "On solving matrix games with pay-offs of triangular fuzzy numbers: Certain observations and generalizations," European Journal of Operational Research, vol. 246, no. 2, pp. 575-581, 2015.

[12] Y.-W. Chen and M. Larbani, "Two-person zero-sum game approach for fuzzy multiple attribute decision making problems," Fuzzy Sets and Systems, vol. 157, no. 1, pp. 34-51, 2006.

[13] M. Larbani, "Solving bimatrix games with fuzzy payoffs by introducing nature as a third player," Fuzzy Sets and Systems, vol. 160, no. 5, pp. 657-666, 2009.

[14] D.-F. Li, "Linear programming approach to solve intervalvalued matrix games," Omega , vol. 39, no. 6, pp. 655-666, 2011.

[15] D.-F. Li, "A fast approach to compute fuzzy values of matrix games with payoffs of triangular fuzzy numbers," European Journal of Operational Research, vol. 223, no. 2, pp. 421-429, 2012.

[16] L. Cunlin and Z. Qiang, "Nash equilibrium strategy for fuzzy non-cooperative games," Fuzzy Sets and Systems, vol. 176, no. 1, pp. $46-55,2011$.

[17] T. Maeda, "Characterization of the equilibrium strategy of the bimatrix game with fuzzy payoff," Journal of Mathematical Analysis and Applications, vol. 251, no. 2, pp. 885-896, 2000.

[18] T. Maeda, "On characterization of equilibrium strategy of twoperson zero-sum games with fuzzy payoffs," Fuzzy Sets and Systems, vol. 139, no. 2, pp. 283-296, 2003.

[19] J.-X. Nan, M.-J. Zhang, and D.-F. Li, "A methodology for matrix games with payoffs of triangular intuitionistic fuzzy number," Journal of Intelligent \& Fuzzy Systems: Applications in Engineering and Technology, vol. 26, no. 6, pp. 2899-2912, 2014.

[20] I. C. H. I. R. O. Nishazaki and M. A. S. A. T. S. C. H. I. Sakawa, "Fuzzy and multiobjective games for conflict resolution," Physica-Verlag, 2001.

[21] V. Vijay, S. Chandra, and C. R. Bector, "Matrix games with fuzzy goals and fuzzy payoffs," Omega, vol. 33, no. 5, pp. 425-429, 2005.

[22] C. Tan, Z. Liu, D. D. Wu, and X. Chen, "Cournot game with incomplete information based on rank-dependent utility theory under a fuzzy," International Journal of Production Research, vol. 56, no. 5, pp. 1789-1805, 2018.

[23] J. Gao, "Credibilistic game with fuzzy information," Journal of Uncertain Systems, vol. 1, no. 1, pp. 74-80, 2007.

[24] J. Gao, Z.-Q. Liu, and P. Shen, "On characterization of credibilistic equilibria of fuzzy-payoff two-player zero-sum game," Soft Computing, vol. 13, no. 2, pp. 127-132, 2009.

[25] J. Gao and X. Yang, "Credibilistic bimatrix game with asymmetric information: Bayesian optimistic equilibrium strategy," International Journal of Uncertainty, Fuzziness and KnowledgeBased Systems, vol. 21, no. 1, pp. 89-100, 2013.

[26] C. Tan, Z. Feng, C. Li, and W. Yi, "Optimal Bayesian equilibrium for $\mathrm{n}$-person credibilistic non-cooperative game with risk aversion," Journal of Intelligent \& Fuzzy Systems: Applications in Engineering and Technology, vol. 33, no. 2, pp. 741-751, 2017.

[27] P. Shen and J. Gao, "Coalitional game with fuzzy payoffs and credibilistic core," Soft Computing, vol. 15, no. 4, pp. 781-786, 2010.
[28] P. C. Fishburn and G. A. Kochenberger, "Two-piece von neumann-morgenstern utility functions," Decision Sciences, vol. 10, no. 4, pp. 503-518, 1979.

[29] D. Kahneman and A. Tversky, "Prospect theory: an analysis of decision under risk," Econometrica, vol. 47, no. 2, pp. 263-291, 1979.

[30] R. M. Kramer, "Windows of vulnerability or cognitive illusions? Cognitive processes and the nuclear arms race," Journal of Experimental Social Psychology, vol. 25, no. 1, pp. 79-100, 1989.

[31] S. E. Taylor, "Asymmetrical effects of positive and negative events: The mobilization-minimization hypothesis," Psychological Bulletin, vol. 110, no. 1, pp. 67-85, 1991.

[32] A. Tversky and D. Kahneman, "Advances in prospect theory: cumulative representation of uncertainty," Journal of Risk and Uncertainty, vol. 5, no. 4, pp. 297-323, 1992.

[33] J. Shalev, "Loss aversion equilibrium," International Journal of Game Theory, vol. 29, no. 2, pp. 269-287, 2000.

[34] B. Driesen, A. Perea, and H. Peters, "On loss aversion in bimatrix games," Theory and Decision, vol. 68 , no. 4, pp. 367391, 2010.

[35] B. Driesen, A. Perea, and H. Peters, "The Kalai-Smorodinsky bargaining solution with loss aversion," Mathematical Social Sciences, vol. 61, no. 1, pp. 58-64, 2011.

[36] B. Driesen, A. Perea, and H. Peters, "Alternating offers bargaining with loss aversion," Mathematical Social Sciences, vol. 64, no. 2, pp. 103-118, 2012.

[37] H. Peters, "A preference foundation for constant loss aversion," Journal of Mathematical Economics, vol. 48, no. 1, pp. 21-25, 2012.

[38] J. Shalev, "Loss aversion and bargaining," Theory and Decision. An International Journal for Multidisciplinary Advances in Decision Science, vol. 52, no. 3, pp. 201-232, 2002.

[39] B. Liu, Uncertainty Theory, Springer, Berlin, Germany, 2004.

[40] Y. Chen, Y. Liu, and X. Wu, "A new risk criterion in fuzzy environment and its application," Applied Mathematical Modelling, vol. 36, no. 7, pp. 3007-3028, 2012.

[41] Y. Liu, X. Wu, and F. Hao, "A new chance-variance optimization criterion for portfolio selection in uncertain decision systems," Expert Systems with Applications, vol. 39, no. 7, pp. 6514-6526, 2012.

[42] X. Li, D. Wang, K. Li, and Z. Gao, "A green train scheduling model and fuzzy multi-objective optimization algorithm," Applied Mathematical Modelling, vol. 37, no. 4, pp. 2063-2073, 2013.

[43] L. Yang, K. Li, Z. Gao, and X. Li, "Optimizing trains movement on a railway network," Omega , vol. 40, no. 5, pp. 619-633, 2012.

[44] B. Liu, Uncertainty Theory, Springer-Verlag, Berlin, Germany, 2nd edition, 2004.

[45] H. Prade and D. Dubois, Fuzzy sets and systems, Theory and applications, New York, NY, USA, 1980.

[46] L. A. Zadeh, "Fuzzy sets," Information and Computation, vol. 8, pp. 338-353, 1965.

[47] C. Negoita, L. Zadeh, and H. Zimmermann, "Fuzzy sets as a basis for a theory of possibility," Fuzzy Sets and Systems, vol. 1, no. 3-28, pp. 61-72, 1978.

[48] A. Nagoor Gani and S. N. Mohamed Assarudeen, "A new operation on triangular fuzzy number for solving fuzzy linear programming problem," Applied Mathematical Sciences, vol. 6, no. $9-12$, pp. 525-532, 2012. 
[49] B. Liu and Y.-K. Liu, "Expected value of fuzzy variable and fuzzy expected value models," IEEE Transactions on Fuzzy Systems, vol. 10, no. 4, pp. 445-450, 2002.

[50] Y. Liu and B. Liu, "Expected value operator of random fuzzy variable and randomfuzzy expected value models," Int. J. Uncertain. Fuzziness Knowledge-Based Systems, vol. 11, no. 2, pp. 195215, 2003.

[51] B. Liu, Theory and Practice of Uncertain Programming, PhysicaVerlag, Heidelberg, Germany, 2002. 


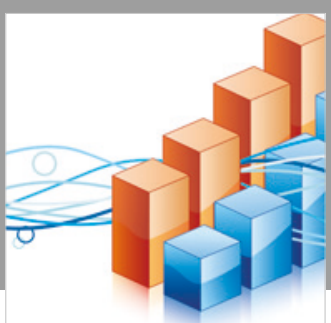

Advances in

Operations Research

\section{-n-m}
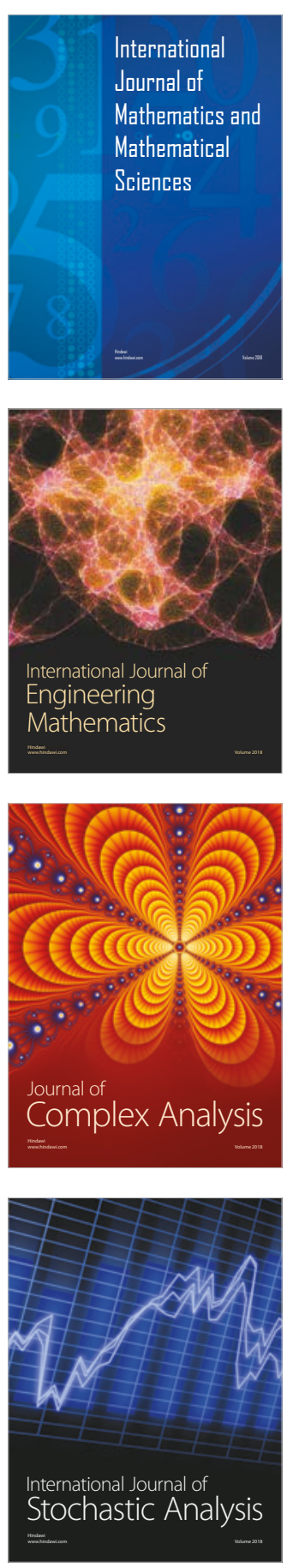
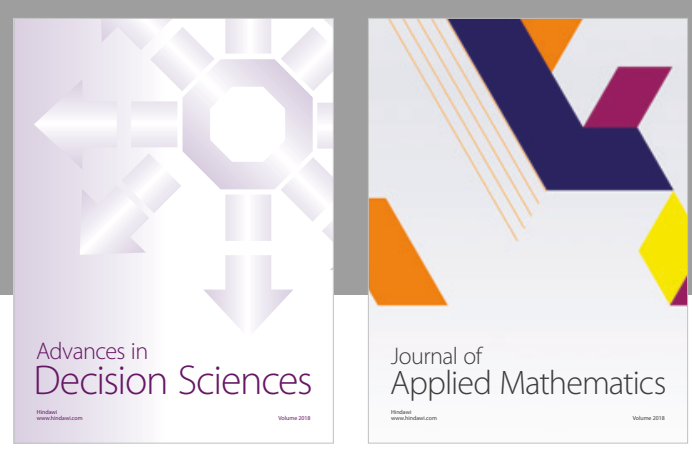

Journal of

Applied Mathematics
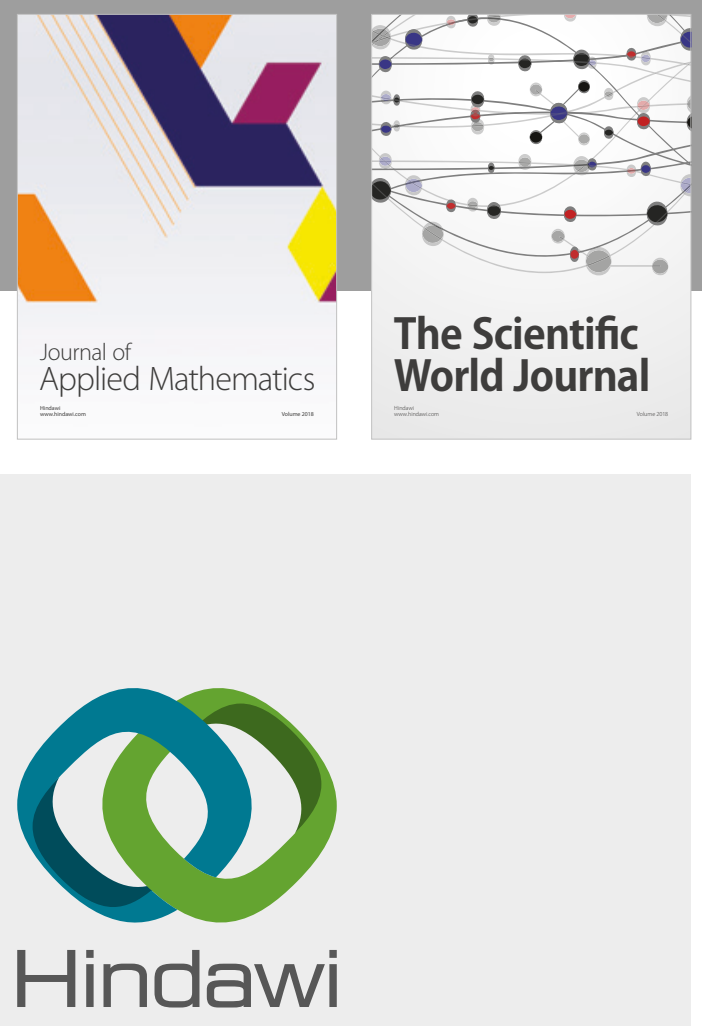

Submit your manuscripts at

www.hindawi.com

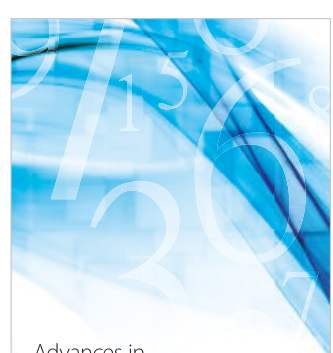

Advances in
Numerical Analysis
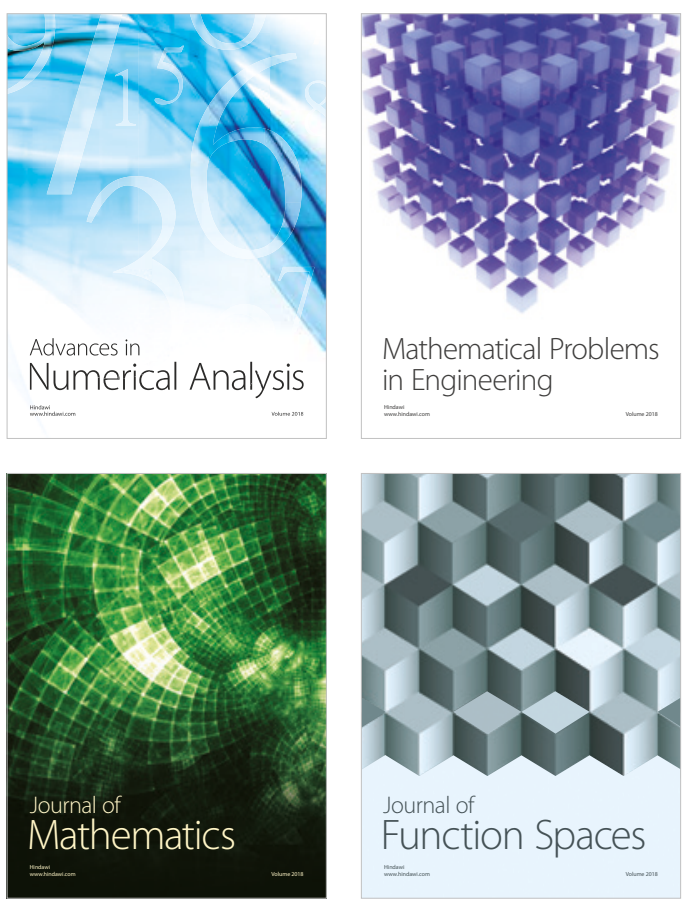

Mathematical Problems in Engineering

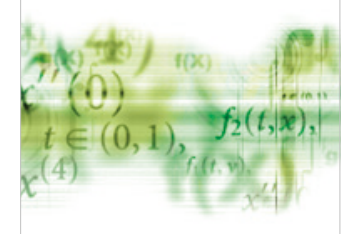

International Journal of

Differential Equations

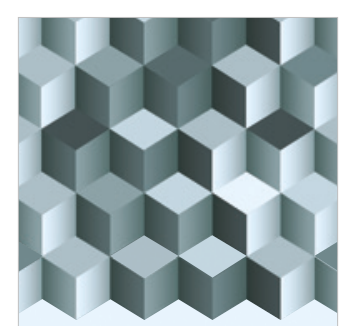

Journal of

Function Spaces
The Scientific

World Journal

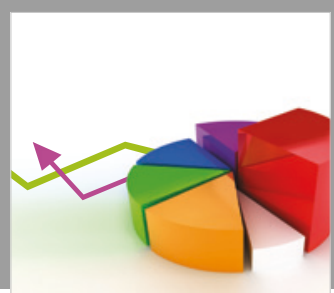

Journal of

Probability and Statistics
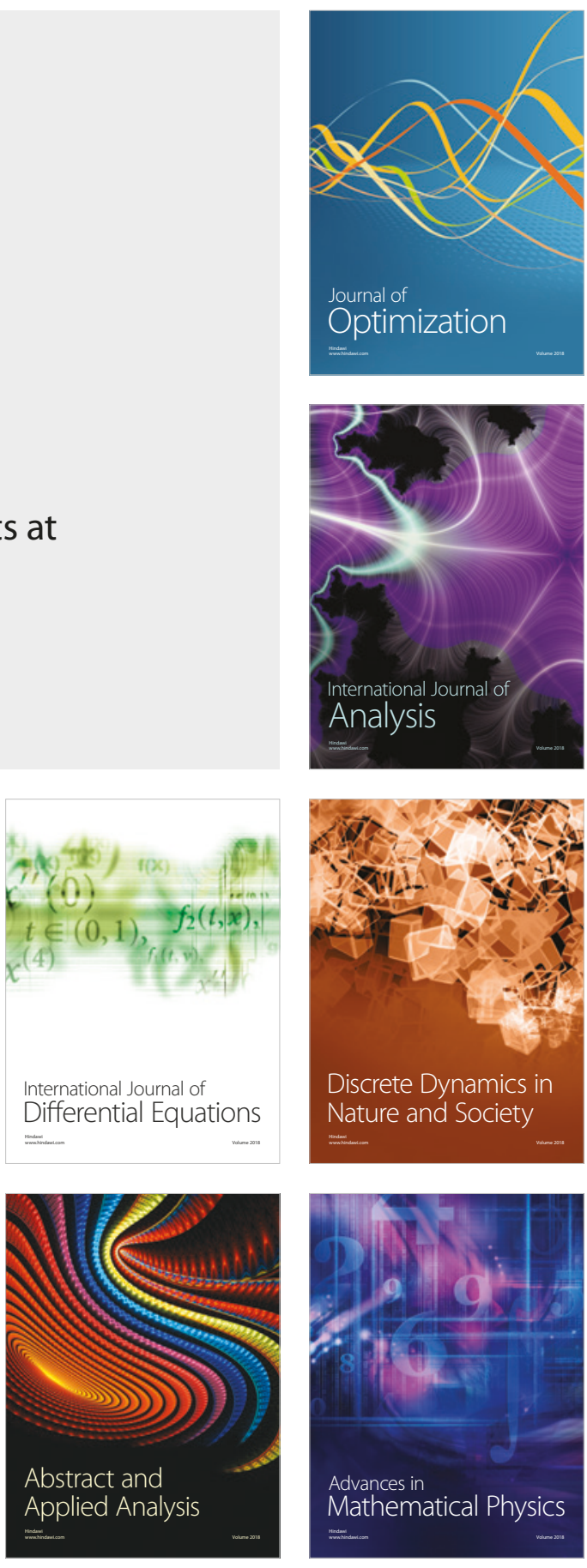